\title{
A STRUCTURE THEOREM FOR 2-STRETCHED GORENSTEIN ALGEBRAS
}

\author{
GIANFRANCO CASNATI AND ROBERTO NOTARI
}

\begin{abstract}
In this paper, we study isomorphism classes of local, Artinian, Gorenstein $k$-algebras $A$ whose maximal ideal $\mathfrak{M}$ satisfies $\operatorname{dim}_{k}\left(\mathfrak{M}^{3} / \mathfrak{M}^{4}\right)=1$ by means of Macaulay's inverse system generalizing a recent result by Elias and Rossi. Then we use such results in order to complete the description of the singular locus of the Gorenstein locus of $\mathcal{H} i l b_{11}\left(\mathbb{P}_{k}^{n}\right)$.
\end{abstract}

1. Introduction and notation. Throughout this paper, a $k$ algebra is an associative, commutative and unitary algebra over an algebraically closed field $k$ of characteristic 0 .

The study of Artinian $k$-algebras is a classical topic in commutative algebra. It is well known that each Artinian $k$-algebra is a direct sum of local ones; hence, one can restrict attention to the local case.

Two important invariants of each local, Artinian $k$-algebra $A$ are its dimension $d:=\operatorname{dim}_{k}(A)$ as $k$-vector space and the Hilbert function $H_{A}$ of $A$, i.e., the Hilbert function of the associated graded ring

$$
\operatorname{gr}(A):=\bigoplus_{i=0}^{\infty} \mathfrak{M}^{i} / \mathfrak{M}^{i+1},
$$

$\mathfrak{M}$ being the maximal ideal of $A$.

When $d \leq 6$, some authors classified such algebras, $A$, in terms of $H_{A}$, e.g., (see $[\mathbf{1 6}, \mathbf{1 7}, \mathbf{1 9}]$ ). As $d$ increases, the picture becomes much more complicated and not easy to handle with the same methods (see

2010 AMS Mathematics subject classification. Primary 13H10, Secondary $13 \mathrm{H} 15,14 \mathrm{C} 05$.

Keywords and phrases. Local, Artinian, Gorenstein algebras, Macaulay's correspondence, punctual Hilbert scheme.

Both authors are members of the GNSAGA group of INdAM. They have been supported by the framework of PRIN 2010-2011 'Geometria sulle varietà algebriche,' cofinanced by MIUR.

Received by the editors on January 20, 2014. 
$[\mathbf{1}, \mathbf{1 8}]$ and the references therein), unless we introduce some extra technical hypothesis, e.g., if we restrict to Gorenstein algebras, then a complete classification in terms of $H_{A}$ is available up to $d \leq 9$ (see [2]). However, once more, as $d$ increases, it is not possible to achieve the complete classification of such algebras. For example, it is not possible to classify in the above sense algebras $A$ with $H_{A}=(1,4,4,1)$. At the same time, researchers have focused on some interesting classes of local, Artinian, Gorenstein $k$-algebras (see $[\mathbf{7}, \mathbf{8}, \mathbf{9}, \mathbf{1 0}, \mathbf{1 3}, \mathbf{2 0}]$ ).

Nevertheless, it is possible to prove a general structure result making use of Macaulay's correspondence. Each local, Artinian, Gorenstein $k$ algebra $A$ can be represented as a quotient of the form $k\left[\left[x_{1}, \ldots, x_{n}\right]\right] / J$ for a suitable ideal $J \subseteq\left(x_{1}, \ldots, x_{n}\right)^{2}$. If we look at $k\left[\left[x_{1}, \ldots, x_{n}\right]\right]$ as acting on $k\left[y_{1}, \ldots, y_{n}\right]$ via derivation, i.e., we identify $x_{i}$ with $\partial / \partial y_{i}$, $i=1, \ldots, n$, then $J=\operatorname{Ann}(F)$ for a suitable $F \in k\left[y_{1}, \ldots, y_{n}\right]$ whose degree $s$ is exactly the maximum integer $s$ such that $\mathfrak{M}^{s} \neq 0$, the so called socle degree of $A, \operatorname{sdeg}(A)$.

The main result of [7] is that such an $F$ can be chosen to be homogeneous when the algebra $A$ satisfies $H_{A}=(1, n, n, 1)$. Hence, the classification of such algebras is actually strictly related to the classification up to projectivities of cubic hypersurfaces in $\mathbb{P}_{k}^{n-1}$.

In the present paper, we extend such a result to Artinian, Gorenstein algebras $A$ with $H_{A}=(1, n, m, 1, \ldots)$ in Section 4 , proving the following theorem (see Theorem 4.3).

Theorem A. Let $A$ be a local, Artinian, Gorenstein algebra. Then $n=H_{A}(1), m=H_{A}(2), 1=H_{A}(3)=\cdots=H_{A}(s), s=\operatorname{sdeg}(A)$ if and only if

$$
A \cong k\left[\left[x_{1}, \ldots, x_{n}\right]\right] / \operatorname{Ann}(F),
$$

where $F:=y_{1}^{s}+F_{3}+\sum_{j=m+1}^{n} y_{j}^{2}, F_{3} \in k\left[y_{1}, \ldots, y_{m}\right]$ is a cubic form, $x_{1}^{2} \circ F_{3}=0$ and $x_{2} \circ F_{3}, \ldots, x_{m} \circ F_{3}$ are linearly independent.

Besides the intrinsic interest of the description of local, Artinian algebras of dimension $d$, their study is also important for the characterization of the singular locus of the Hilbert scheme $\mathcal{H i l b}_{d}\left(\mathbb{P}_{k}^{n}\right)$. In some recent papers (see [3], for the case $d \leq 9$ and [4] for the case $d=10$ ) we dealt with such a problem, restricting our attention to the Gorenstein locus $\mathcal{H} i l b_{d}^{G}\left(\mathbb{P}_{k}^{n}\right) \subseteq \mathcal{H} i l b_{d}\left(\mathbb{P}_{k}^{n}\right)$, i.e., the locus of schemes 
$X \cong \operatorname{spec}(A)$ where $A$ is a Gorenstein algebra. In particular, in [4], the structure theorem of [7] has been a helpful tool for such a description.

In [5], a similar analysis has been carried out in the case $d=11$. In that paper we were also able to deal with the singular nature of all $X \in \mathcal{H i l b} b_{11}^{G}\left(\mathbb{P}_{k}^{n}\right)$, but those isomorphic to $\operatorname{spec}(A)$ where $A$ is a local, Artinian, Gorenstein algebra with $H_{A}=(1,4,4,1,1)$. The second main result of the present paper is the complete description of such a kind of $X$ from the following viewpoint. Such a description rests on Theorem A above. The main results of Section 5 can be summarized in the following theorem.

Theorem B. Let $A$ be a local, Artinian, Gorenstein algebra with $H_{A}=(1,4,4,1,1)$. Then $X:=\operatorname{spec}(A) \in \mathcal{H}_{i l b}^{G}\left(\mathbb{P}_{k}^{n}\right)$ is obstructed if, and only if, $A \cong S[4] / \operatorname{Ann}(F)$ where $F$ is in the following list:

(i) $y_{1}^{4}+y_{1}\left(b_{0} y_{2}^{2}+b_{2} y_{3}^{2}+b_{5} y_{4}^{2}\right)+y_{2}^{3}+y_{3}^{3}+y_{4}^{3}, b_{0}, b_{2}, b_{5} \in k$;

(ii) $y_{1}^{4}+y_{1}\left(b_{0} y_{2}^{2}+b_{2} y_{3}^{2}+2 b_{4} y_{3} y_{4}\right)+y_{2}^{3}+y_{3}^{2} y_{4}, b_{0}, b_{2}, b_{4} \in k$;

(iii) $y_{1}^{4}+y_{1}\left(b_{0} y_{2}^{2}+2 b_{1} y_{2} y_{3}+b_{2} y_{3}^{2}+2 b_{0} y_{3} y_{4}\right)+y_{2}^{2} y_{3}+y_{3}^{2} y_{4}, b_{0}, b_{2}, b_{3} \in k$;

(iv) $y_{1}^{4}+y_{1}\left(b_{0} y_{2}^{2}+2 b_{1} y_{2} y_{3}+b_{2} y_{3}^{2}+2 b_{3} y_{2} y_{4}+2 b_{4} y_{3} y_{4}+b_{5} y_{4}^{2}\right)+y_{3}^{2} y_{4}-$ $y_{3}^{2} y_{4}, b_{0}, \ldots, b_{5} \in k,-b_{1}^{2}+b_{0} b_{2}-b_{1} b_{3}-b_{3}^{2}+b_{0} b_{4}+b_{0} b_{5}=0$, $\left(b_{0}, b_{1}, b_{3}\right) \neq(0,0,0)$;

(v) $y_{1}^{4}+y_{1}\left(b_{0} y_{2}^{2}+2 b_{1} y_{2} y_{3}+b_{2} y_{3}^{2}+2 b_{3} y_{2} y_{4}+2 b_{4} y_{3} y_{4}+b_{5} y_{4}^{2}\right)+y_{3}^{2} y_{4}$, $b_{0}, \ldots, b_{5} \in k, b_{1}^{2}-b_{0} b_{2}=0,\left(b_{0}, b_{1}, b_{3}\right) \neq(0,0,0)$

(vi) $y_{1}^{4}+y_{1}\left(b_{0} y_{2}^{2}+2 b_{1} y_{2} y_{3}+b_{2} y_{3}^{2}+2 b_{3} y_{2} y_{4}+2 b_{4} y_{3} y_{4}+b_{5} y_{4}^{2}\right)+y_{3}^{3}$, $b_{0}, \ldots, b_{5} \in k$ and $\operatorname{rk}\left(M_{b}\right) \geq 2$, where

$$
M_{b}:=\left(\begin{array}{lll}
b_{0} & b_{1} & b_{3} \\
b_{1} & b_{2} & b_{4} \\
b_{3} & b_{4} & b_{5}
\end{array}\right) ;
$$

(vii) $y_{1}^{4}+y_{1}\left(b_{0} y_{2}^{2}+2 b_{1} y_{2} y_{3}+b_{2} y_{3}^{2}+2 b_{3} y_{2} y_{4}+2 b_{4} y_{3} y_{4}+b_{5} y_{4}^{2}\right), b_{0}, \ldots, b_{5} \in$ $k$ and $\operatorname{rk}\left(M_{b}\right)=3$, where $M_{b}$ is the matrix above.

1.1. Notation. In what follows, $k$ is an algebraically closed field of characteristic 0 . A $k$-algebra is an associative, commutative and unitary algebra over $k$. For each $N \in \mathbb{N}$, we set $S[N]:=k\left[\left[x_{1}, \ldots, x_{N}\right]\right]$ and $P[N]:=k\left[y_{1}, \ldots, y_{N}\right]$. We denote by $S[N]_{q}$ (respectively, $P[N]_{q}$ ) the homogeneous component of degree $q$ of such a graded $k$-algebra. Let $S[N]_{\leq q}:=\bigoplus_{i=1}^{q} S[N]_{i}$ (respectively, $\left.P[N]_{\leq q}:=\bigoplus_{i=1}^{q} P[N]_{i}\right)$. Finally, 
we set $S[N]_{+}:=\left(x_{1}, \ldots, x_{N}\right) \subseteq S[N]$ to be the unique maximal ideal of $S[N]$.

A local ring $R$ is Gorenstein if its injective dimension as $R$-module is finite. An arbitrary ring $R$ is called Gorenstein if $R_{\mathfrak{M}}$ is Gorenstein for every maximal ideal $\mathfrak{M} \subseteq R$. A scheme $X$ is Gorenstein if and only if, for each point $x \in X$, the ring $\mathcal{O}_{X, x}$ is Gorenstein.

For each numerical polynomial $p(t) \in \mathbb{Q}[t]$, we denote by $\mathcal{H} i l b_{p(t)}\left(\mathbb{P}_{k}^{N}\right)$ the Hilbert scheme of closed subschemes of $\mathbb{P}_{k}^{N}$ with Hilbert polynomial $p(t)$. With abuse of notation, we will denote by the same symbol both a point in $\mathcal{H i l b} b_{p(t)}\left(\mathbb{P}_{k}^{N}\right)$ and the corresponding subscheme of $\mathbb{P}_{k}^{N}$. We denote by $\mathcal{H i l b}_{p(t)}^{G}\left(\mathbb{P}_{k}^{N}\right)$ the locus of points representing Gorenstein schemes.

If $\gamma:=\left(\gamma_{1}, \ldots, \gamma_{N}\right) \in \mathbb{N}^{N}$ is a multi-index, then we set $t^{\gamma}:=$ $t_{1}^{\gamma_{1}} \cdots t_{N}^{\gamma_{N}} \in k\left[t_{1}, \ldots, t_{N}\right]$

For all other notation and results we refer to [12].

2. Some facts on Macaulay's correspondence. Let $A$ be a local, Artinian $k$-algebra with maximal ideal $\mathfrak{M}$. We know that

$$
A \cong S[n] / J
$$

for a suitable ideal $J \subseteq S[n]_{+}^{2} \subseteq S[n]$, where $n:=H_{A}(1)$. Recall that the socle degree $\operatorname{sdeg}(A)$ of $A$ is the greatest integer $s$ such that $\mathfrak{M}^{s} \neq 0$.

For a quick list of results about Macaulay's theory of the inverse system, see e.g., $[\mathbf{1 1}, \mathbf{1 4}]$ and the references therein.

The ring $P[n]$ has a natural structure of $S[n]$-module by identifying $x_{i}$ with $\partial / \partial y_{i}$. Hence,

$$
x^{\alpha} \circ y^{\beta}:= \begin{cases}\alpha !\left(\begin{array}{l}
\beta \\
\alpha
\end{array}\right) y^{\beta-\alpha} & \text { if } \beta \geq \alpha, \\
0 & \text { if } \beta \geq \alpha .\end{cases}
$$

Given an ideal $J \subseteq S[n]$ and an $S[n]$-submodule $M \subseteq P[n]$, we set

$$
\begin{aligned}
J^{\perp} & :=\{F \in P[n] \mid g \circ F=0, \quad \text { for all } g \in J\}, \\
\operatorname{Ann}(M) & :=\{g \in S[n] \mid g \circ F=0, \quad \text { for all } F \in M\} .
\end{aligned}
$$

The main point of Macaulay's theory of the inverse system is that the constructions $J \mapsto J^{\perp}$ and $M \mapsto \operatorname{Ann}(M)$ give rise to a reversing 
inclusion bijection between ideals $J \subseteq S[n]$ such that $S[n] / J$ is a local, Artinian $k$-algebra and finitely generated $S[n]$-submodules $M \subseteq P[n]$.

Gorenstein algebras $A$ with $\operatorname{sdeg}(A)=s$ correspond to cyclic $S[n]$ submodules $\langle F\rangle_{S[n]} \subseteq P[n]$ generated by a polynomial $F$ of degree $s$ in such a bijection.

On the one hand, for each $S[n]$-module $M$, we define

$$
\operatorname{tdf}(M):=\bigoplus_{q=0}^{\infty} \operatorname{tdf}(M)_{q},
$$

where

$$
\operatorname{tdf}(M)_{q}:=\frac{M \cap P[n]_{\leq q}+P[n]_{\leq q-1}}{P[n]_{\leq q-1}} .
$$

The module $\operatorname{tdf}(M)$ can be interpreted as the $S[n]$-submodule of $P[n]$ generated by the top degree forms of all the polynomials in $M$.

On the other hand, for each $f \in S[n]$, the lowest degree of monomials appearing with non-zero coefficient in the minimal representation of $f$ is called the order of $f$, and it is denoted by $\operatorname{ord}(f)$. If

$$
f=\sum_{i=\operatorname{ord}(f)}^{\infty} f_{i}, \quad f_{i} \in S[n]_{i},
$$

then $f_{\text {ord }(f)}$ is called the lowest degree form of $f$. We will denote it by $\operatorname{ldf}(f)$.

Recall that we are assuming $J \subseteq S[n]_{+}^{2}$; thus, ord $(f) \geq 2$ for each $f \in J$. The lowest degree form ideal $\operatorname{ldf}(J)$ associated to $J$ is

$$
\operatorname{ldf}(J):=(\operatorname{ldf}(f) \mid f \in J) \subseteq S[n] .
$$

We have $\operatorname{ldf}(\operatorname{Ann}(M))=\operatorname{Ann}(\operatorname{tdf}(M))$ (see [11]; see also [7, formulas (2), (3)]). As an immediate consequence, we have

$$
\operatorname{gr}(S[n] / \operatorname{Ann}(M)) \cong S[n] / \operatorname{ldf}(\operatorname{Ann}(M)) \cong S[n] / \operatorname{Ann}(\operatorname{tdf}(M)) .
$$

Thus,

$$
H_{S[n] / \operatorname{Ann}(M)}(q)=\operatorname{dim}_{k}\left(\operatorname{tdf}(M)_{q}\right) .
$$

The module $M$ is non-degenerate if

$$
H_{S[n] / \operatorname{Ann}(M)}(1)=\operatorname{dim}_{k}\left(\operatorname{tdf}(M)_{1}\right)=n .
$$


In other words, $M$ is non-degenerate if and only if the classes of $y_{1}, \ldots, y_{n}$ are in $\operatorname{tdf}(M)$.

In what follows, in order to simplify the notation, we will write $\operatorname{tdf}(F)$ and $\operatorname{Ann}(F)$ instead of $\operatorname{tdf}\left(\langle F\rangle_{S[n]}\right)$ and $\operatorname{Ann}\left(\langle F\rangle_{S[n]}\right)$, respectively.

Assume that $A$ is Gorenstein with $s:=\operatorname{sdeg}(A)$. Thus, $\operatorname{Soc}(A)=$ $\mathfrak{M}^{s} \cong k$. In particular, there exists

$$
F:=\sum_{i=0}^{s} F_{i}, \quad F_{i} \in P[n]_{i},
$$

such that $A \cong S[n] / \operatorname{Ann}(F)$. For each $h \geq 0$, we set

$$
F_{\geq h}:=\sum_{i=h}^{s} F_{i}
$$

(hence, $F_{s}=F_{\geq s}$ ).

We can always assume that $F_{0}=0$. It is easy to check that $\operatorname{Ann}(F)=\operatorname{Ann}(F+\sigma \circ F)$ for every $\sigma \in S[n]$. The following lemma allows us to find a simpler polynomial $\widehat{F}$ such that $\operatorname{Ann}(\widehat{F})=\operatorname{Ann}(F)$. For its proof, see [5, Lemma 2.1].

Lemma 2.1. Let $F, \widehat{F} \in P[n]$ be such that $F-\widehat{F} \in P[n]_{\leq 1}$. If $\operatorname{Ann}(F) \subseteq S[n]_{+}^{2}$, then $\operatorname{Ann}(F)=\operatorname{Ann}(\widehat{F})$.

In particular, if $J \subseteq S[n]_{+}^{2}$, then we can assume that $J=\operatorname{Ann}(F)$ where $F=F_{\geq 2}$. We do make such an assumption in what follows.

We have a filtration with proper ideals (see [14]) of $\operatorname{gr}(A) \cong$ $S[n] / \operatorname{ldf}(\operatorname{Ann}(F))$

$$
\begin{aligned}
C_{A}(0):=\operatorname{gr}(A) & \supset C_{A}(1) \supseteq C_{A}(2) \supseteq \cdots \\
& \supseteq C_{A}(s-2) \supseteq C_{A}(s-1):=0 .
\end{aligned}
$$

Via the epimorphism $S[n] \rightarrow \operatorname{gr}(A)$, we obtain an induced filtration

$$
\begin{aligned}
\widehat{C}_{A}(0):=S[n] & \supset \widehat{C}_{A}(1) \supseteq \widehat{C}_{A}(2) \supseteq \cdots \\
& \supseteq \widehat{C}_{A}(s-2) \supseteq \widehat{C}_{A}(s-1):=\operatorname{ldf}(\operatorname{Ann}(F)) .
\end{aligned}
$$


The quotients $Q_{A}(a):=C_{A}(a) / C_{A}(a+1) \cong \widehat{C}_{A}(a) / \widehat{C}_{A}(a+1)$ are reflexive graded $\operatorname{gr}(A)$-modules whose Hilbert function is symmetric around $(s-a) / 2$. In general, $\operatorname{gr}(A)$ is no longer Gorenstein, but the first quotient

$$
G(A):=Q_{A}(0) \cong S[n] / \operatorname{Ann}\left(F_{s}\right)
$$

is characterized by the property of being the unique (up to isomorphism) graded Gorenstein quotient $k$-algebra of $\operatorname{gr}(A)$ with the same socle degree. The Hilbert function of $A$ satisfies

$$
H_{A}(i)=H_{\operatorname{gr}(A)}(i)=\sum_{a=0}^{s-2} H_{Q_{A}(a)}(i), \quad i \geq 0 .
$$

Since $H_{A}(0)=H_{G(A)}(0)=1$, it follows that, if $a \geq 1$, then $Q_{A}(a)_{0}=0$, whence $Q_{A}(a)_{i}=0$ when $i \geq s-a$ (see [14]) for the same values of $a$.

Moreover,

$$
H_{\operatorname{gr}(A) / C_{A}(a+1)}(i)=H_{S[n] / \widehat{C}_{A}(a+1)}(i)=\sum_{\alpha=0}^{a} H_{Q_{A}(\alpha)}(i), \quad i \geq 0 .
$$

We set

$$
f_{h}:=\sum_{\alpha=0}^{s-h} H_{Q_{A}(\alpha)}(1)=H_{S[n] / \widehat{C}_{A}(s-h+1)}(1)=H_{\operatorname{gr}(A) / C_{A}(s-h+1)}(1)
$$

(so that $n=H_{A}(1)=f_{2}$ ).

We recall that a local, Artinian $k$-algebra $A$ is $c$-stretched for some positive integer $c$ if $c$ is the maximum positive integer $j$, if any, such that $H_{A}(j)>1$. If $H_{A}(j) \leq 1$ for every $j$, we say that $A$ is 0 -stretched. Finally, if $c \leq 1$, we simply say that $A$ is stretched.

We recall some facts about $c$-stretched algebras $A$. Trivially, when $c=0$, then $A \cong S[1] /\left(x_{1}^{s+1}\right)$ (recall that $S[1]:=k\left[\left[x_{1}\right]\right]$ ). Stretched algebras have been completely classified in [20], with particular attention to the Gorenstein case.

There are many results about 2-stretched Gorenstein algebras. A complete description of such algebras when $\operatorname{sdeg}(A)=3$ can be found in [7]. 2-stretched algebras with $H_{A}(2)=2$ have been examined in several papers (see, e.g., $[\mathbf{3}, \mathbf{1 0}])$. A complete classification in the case 
$\operatorname{sdeg}(A) \geq 4$ and $H_{A}(2)=3$ can be found in [3]. Some very partial results are known when $\operatorname{sdeg}(A)=4$ and $H_{A}(2)=4$ (see [4]).

3. On the homogeneous summands of the apolar polynomial. Let $A \cong S[n] / J$ be an Artinian, Gorenstein $k$-algebra where $J=\operatorname{Ann}(F)$ for a suitable $F=F_{\geq 2} \in P[n]$. Such a polynomial strongly depends on the representation of $A$ as a quotient of $S[n]$.

For the reader's benefit, we recall (see [14, Theorems 5.3A, 5.3B]) in this section that it is always possible to choose a system of generators of $S[n]_{+}$such that $F$ satisfies $F_{i} \in P\left[f_{i}\right]$. If $A \cong S[n] / \operatorname{Ann}(F)$, then such a property is not automatically satisfied by $F$, due to the possible existence of exotic summands in the homogeneous decomposition of $F$ as the following well-known example shows.

Example 3.1. Let $J:=\left(x_{2}^{2}, 20 x_{1}^{2} x_{2}-x_{1}^{4}\right) \subseteq S[2]$, then $J^{\perp}=\langle F\rangle_{S[2]}$, where $F:=y_{1}^{5}+y_{1}^{3} y_{2}$. In particular,

$$
F_{5}=y_{1}^{5}, \quad F_{4}=y_{1}^{3} y_{2}, \quad F_{3}=F_{2}=0 .
$$

We have $\operatorname{tdf}(F)_{5}=\left\langle y_{1}^{5}\right\rangle, \operatorname{tdf}(F)_{4}=\left\langle y_{1}^{4}\right\rangle, \operatorname{tdf}(F)_{3}=\left\langle y_{1}^{3}\right\rangle, \operatorname{tdf}(F)_{2}=$ $\left\langle y_{1}^{2}, y_{1} y_{2}\right\rangle, \operatorname{tdf}(F)_{1}=\left\langle y_{1}, y_{2}\right\rangle$; thus, $H_{A}=(1,2,2,1,1,1)$ due to relation (2.1). Hence, we have $H_{G(A)}=(1,1,1,1,1,1), H_{Q(1)}=(0,0,0,0,0,0)$ and $H_{Q(2)}=(0,1,1,0,0,0)$. In particular, $F_{4} \notin P\left[f_{4}\right]$ because $f_{4}=1$ in our case.

Proposition 3.2. Let $A$ be a local, Artinian, Gorenstein k-algebra. If $n:=H_{A}(1)$ and $s:=\operatorname{sdeg}(A)$, then

$$
A \cong S[n] / \operatorname{Ann}(F),
$$

where

$$
F:=\sum_{i=2}^{s} F_{i}+\sum_{j=f_{3}+1}^{n} y_{j}^{2}, \quad F_{i} \in P\left[f_{i}\right]_{i}, i \geq 3, \quad F_{2} \in P\left[f_{3}\right]_{2} .
$$

Proof. Due to the aforementioned Theorems 5.3A and 5.3B of [14] (see also the thesis [15]; in particular, see Theorem 4.38 where an expanded version of the proof is provided), we know the existence of a representation $A \cong S[n] / \operatorname{Ann}\left(\sum_{i=2}^{s} F_{i}\right)$ with $F_{i} \in P\left[f_{i}\right]_{i}$. Now we 
prove that $F_{2}$ can actually be written as the sum of an element in $P\left[f_{3}\right]$ plus $\sum_{f_{3}+1}^{n} y_{i}^{2}$.

First, we prove that we can make a suitable linear change of $y_{1}, \ldots, y_{n}$ in such a way that the linear space generated by $y_{1}, \ldots, y_{f_{3}}$ remains unchanged and the homogeneous part of degree 2 of $F$ is

$$
F_{2}+\sum_{j=f_{3}+1}^{n} y_{j}^{2}, \quad F_{2} \in P\left[f_{3}\right] .
$$

Up to a suitable linear transformation of the variables $y_{f_{3}+1}, \ldots, y_{n}$, we can assume that such a homogeneous part of $F$ has the form

$$
\sum_{j=f_{3}+1}^{n} \lambda_{j} y_{j}^{2}+Q
$$

where $\lambda_{j} \in\{0,1\}$ and

$$
Q=\sum_{i=1}^{f_{3}} \sum_{j=i}^{n} q_{i, j} y_{i} y_{j} .
$$

Since $H_{A}(1)=n$, we know that the classes of $y_{1}, \ldots, y_{n}$ are in $\operatorname{tdf}(F)$. It follows that we have relations of the form:

$$
\begin{aligned}
& \sum_{i=1}^{n} u_{i}\left(x_{i} \circ F\right)+\text { linear combination of derivatives of } F \\
& \text { of order at least } 2=y_{j}+\text { constant. }
\end{aligned}
$$

Since $F_{i} \in P\left[f_{i}\right]_{i} \subseteq P\left[f_{3}\right]_{i}, i \geq 3$, we deduce $x_{j} \circ F=2 \lambda_{j} y_{j}+x_{j} \circ Q$.

The only derivatives of $F$ of degree $s-1$ are $x_{j} \circ F, j=1, \ldots, f_{s-1}$. They are linearly independent because $\operatorname{dim}_{k}\left(\operatorname{tdf}(F)_{s-1}\right)=H_{A}(s-1)=$ $f_{s-1}$. It follows that $u_{j}=0$, for all $j=1, \ldots, f_{s}$. Since no derivatives of order 2 contain $y_{j}$, for $j \geq f_{3}+1$ (recall that $F_{i} \in P\left[f_{3}\right]_{i}, i \geq 3$ ), it also follows that the linear combination of such derivatives in the first member of the above equality must be a constant. We conclude that

$$
\sum_{i=f_{3}+1}^{n} u_{i}\left(x_{i} \circ F\right)=y_{j}+\text { constant. }
$$


We deduce that necessarily $\lambda_{j}=1$, for $j \geq f_{3}+1$ (recall that $\lambda_{j} \in\{0,1\}$ and $\left.x_{j} \circ Q \in P\left[f_{3}\right]\right)$. Up to a suitable linear transformation of the variables $y_{1}, \ldots, y_{n}$, fixing $y_{1}, \ldots, y_{f_{3}}$, we can finally assume that $F_{2}:=Q \in P\left[f_{3}\right]_{2}$.

The proof of the statement is complete.

4. The structure theorem. Now we turn our attention to algebras $A$ with $H_{A}=(1, n, m, 1, \ldots, 1)$. We first prove the following preparatory lemma improving Proposition 3.2 in such a particular case.

Lemma 4.1. Let $A$ be a local, Artinian, Gorenstein 2-stretched $k$ algebra. If $n:=H_{A}(1), m:=H_{A}(2), s:=\operatorname{sdeg}(A)$, then

$$
A \cong S[n] / \operatorname{Ann}(F) \text {, }
$$

where

$$
F:=y_{1}^{s}+F_{3}+F_{2}+\sum_{j=m+1}^{n} y_{j}^{2}, \quad F_{i} \in P[m]_{i},
$$

$x_{1}^{2} \circ F_{3}=x_{1}^{2} \circ F_{2}=0$ and $x_{2} \circ F_{3}, \ldots, x_{m} \circ F_{3}$ are linearly independent.

Proof. If $m=1$, in particular, if $n=1$, then the statement is trivial. Thus we can assume $n, m \geq 2$, whence $s \geq 3$.

If $s=3$, then $F=\widetilde{F}_{3}+F_{2}$. Up to a linear change of variables in $S[n]$, we can assume that the coefficient of $y_{1}^{3}$ in $\widetilde{F}_{3}$ is 1 , so that $\widetilde{F}_{3}=y_{1}^{3}+F_{3}$ with $x_{1}^{3} \circ F_{3}=0$. If the coefficient of $y_{1}^{2}$ in $F_{3}$ is $\ell \in k\left[y_{2}, \ldots, y_{n}\right]$, then the substitution $y_{1} \mapsto y_{1}-\ell / 3$ finally gives the condition $x_{1}^{2} \circ F_{3}=0$.

From now on, let $s \geq 4$. Equality (2.3) and the symmetry of $H_{Q_{A}(a)}$ around $(s-a) / 2$ imply $H_{Q_{A}(a)}=0$ when $i=1, \ldots, s-5$. For the same reason we also know that $H_{Q_{A}(s-4)}(i)=0$ if $i \neq 2$. We thus have (see equality (2.4))

$$
H_{S[n] / \widehat{C}_{A}(s-3)}=\sum_{\alpha=0}^{s-4} H_{Q_{A}(\alpha)}=H_{Q_{A}(0)}+H_{Q_{A}(s-4)} .
$$

If $H_{Q_{A}(s-4)}(2)=p \neq 0$, then $H_{S[n] / \widehat{C}_{A}(s-3)}=(1,1,1+p, 1, \ldots, 1)$ which is not possible because the quotient $S[n] / \widehat{C}_{A}(s-3)$ is generated in degree 1 as a $k$-algebra. Thus, the only possible symmetric 
decomposition of $H_{A}$ is

$$
H_{Q_{A}(a)}= \begin{cases}(1,1,1,1, \ldots, 1) & \text { if } a=0, \\ (0,0,0,0, \ldots, 0) & \text { if } a=1, \ldots, s-4, \\ (0, m-1, m-1,0, \ldots, 0) & \text { if } a=s-3 \\ (0, n-m, 0,0, \ldots, 0) & \text { if } a=s-2 .\end{cases}
$$

It follows that $f_{2}=n, f_{3}=m$ and $f_{4}=\cdots=f_{s}=1$. Due to Proposition 3.2 there exists an isomorphism $A \cong S[n] / \operatorname{Ann}(F)$, where

$$
F:=\sum_{i=2}^{s} F_{i}+\sum_{j=f_{3}+1}^{n} y_{j}^{2},
$$

$F_{2} \in P[m]_{2}, F_{3} \in P[m]_{3}$ and $F_{i} \in P[1]_{i}, i=4, \ldots, s$. In particular, if $s \geq 4$, then $G(A) \cong S[1] /\left(y_{1}^{s}\right)$; thus, we can assume that $F_{s}=y_{1}^{s}$ due to formula $(2.2)$.

Let $e_{i}$ be the $i$ th canonical generator of $\mathbb{N}^{m}$. For each $s$, let

$$
F_{3}=\sum_{\alpha \in \mathbb{N}^{m},|\alpha|=3} \frac{s !}{\alpha !} u_{\alpha} y^{\alpha},
$$

so that $x^{\alpha}-u_{\alpha} x_{1}^{s} \in \operatorname{Ann}(F)$ when $\alpha \in \mathbb{N}^{m},|\alpha|=3$ and $\alpha \neq 3 e_{1}$.

Let us consider the automorphism $\varphi$ of $S[n]$ defined by

$$
\varphi\left(x_{j}\right)=\widehat{x}_{j}:= \begin{cases}x_{j} & \text { if } j \neq 2, \ldots, m, \\ x_{j}-u_{2 e_{1}+e_{j}} x_{1}^{s-2} & \text { if } j=2, \ldots, m .\end{cases}
$$

We have an isomorphism

$$
S[n] / \varphi^{-1}(\operatorname{Ann}(F)) \cong S[n] / \operatorname{Ann}(F) \cong A .
$$

We conclude the existence of $\widehat{F} \in P[n]$ such that $\varphi^{-1}(\operatorname{Ann}(F))=$ $\operatorname{Ann}(\widehat{F})$. Let

$$
\widehat{F}=\sum_{i=2}^{s} \widehat{F}_{i}
$$

Due to the definition of $\varphi$, we have that $\widehat{x}^{\alpha} \in \operatorname{Ann}(F)$ if either $|\alpha| \geq 4$ and $\alpha \neq|\alpha| e_{1}$, or if $\widehat{x}^{\alpha}$ does not contain $x_{1}, \ldots, x_{m}$ and $|\alpha| \geq 3$, or if $\alpha=2 e_{1}+e_{j}, j=2, \ldots, m$. It follows that $x^{\alpha} \in \varphi^{-1}(\operatorname{Ann}(F))=$ $\operatorname{Ann}(\widehat{F})$ in the same ranges. 
The first condition implies that we can still assume $\widehat{F}_{i} \in P[1]$, $i=4, \ldots, s$. The second one implies that $\widehat{F}_{3} \in P[m]$. The third condition implies $x_{1}^{2} \circ \widehat{F}_{3} \in P[1]_{1}$.

Moreover the automorphism $\varphi$ fixes the subalgebra $S[m] \subseteq S[n]$ and leaves the variables $y_{m+1}, \ldots, y_{n}$ unchanged. Thus, we can still write the homogeneous part of degree 2 of $\widehat{F}$ as

$$
\widehat{F}_{2}+\sum_{j=m+1}^{n} y_{j}^{2}, \quad \widehat{F}_{2} \in P[m]_{2}
$$

as in the statement of Proposition 3.2.

Adding to $\widehat{F}$ a suitable linear combination of the derivatives $x_{1}^{i} \circ \widehat{F}$ we can finally assume that $A \cong S[n] / \operatorname{Ann}(\widehat{F})$ where

$$
\widehat{F}:=y_{1}^{s}+\widehat{F}_{3}+\widehat{F}_{2}+\sum_{j=m+1}^{n} y_{j}^{2},
$$

and $x_{1}^{2} \circ \widehat{F}_{3}=x_{1}^{2} \circ \widehat{F}_{2}=0$.

Finally $\operatorname{tdf}(\widehat{F})_{2}$ is generated by the classes of $y_{1}^{2}$ and $x_{2} \circ \widehat{F}_{3}, \ldots, x_{m} \circ$ $\widehat{F}_{3}$. Since,

$$
\operatorname{dim}\left(\operatorname{tdf}(\widehat{F})_{2}\right)=H_{A}(2)=m,
$$

we conclude that $x_{2} \circ \widehat{F}_{3}, \ldots, x_{m} \circ \widehat{F}_{3}$ are linearly independent.

Remark 4.2. The above lemma can be easily generalized to any local, Artinian, Gorenstein $c$-stretched algebra $A$ for each $c$ as follows. If $n:=H_{A}(1), m:=H_{A}(2)$ and $s:=\operatorname{sdeg}(A)$, then

$$
A \cong S[n] / \operatorname{Ann}(F),
$$

where

$$
F:=y_{1}^{s}+\sum_{i=2}^{c+1} F_{i}+\sum_{j=m+1}^{n} y_{j}^{2}, \quad F_{i} \in P\left[f_{i}\right]_{i}, i \geq 3
$$

$F_{2} \in P\left[f_{3}\right]_{i}, x_{1}^{c} \circ F_{c+1}=x_{1}^{i} \circ F_{i}=0, i=3, \ldots, c+1$ and $x_{2} \circ$ $F_{c+1}, \ldots, x_{m} \circ F_{c+1}$ are linearly independent.

Following the method used to deal with algebras $A$ with $\operatorname{sdeg}(A)=3$ (in [7]) and with compressed algebras (in [8]), we will show how 
to construct, for each local, Artinian, Gorenstein $k$-algebra $A$ with $H_{A}=(1, n, m, 1, \ldots, 1), \operatorname{sdeg}(A)=s$, a distinguished polynomial $F_{3} \in P[m]_{3}$ such that

$$
A \cong S[n] / \operatorname{Ann}\left(y_{1}^{s}+F_{3}+\sum_{j=m+1}^{n} y_{j}^{2}\right) .
$$

Let

$$
F=y_{1}^{s}+F_{3}+F_{2}+\sum_{j=m+1}^{n} y_{j}^{2}
$$

be as in the statement of Lemma 4.1 and set $A:=S[n] / \operatorname{Ann}(F)$. We look for a particular algebra automorphism $\varphi$ of $S[n]$ mapping $\operatorname{Ann}(F)$ to $\operatorname{Ann}\left(F_{\text {simple }}\right)$, where

$$
F_{\text {simple }}:=F-F_{2}=y_{1}^{s}+F_{3}+\sum_{j=m+1}^{n} y_{j}^{2} .
$$

If we set $\varphi\left(x_{i}\right)=z_{i}$, then $z_{1}, \ldots, z_{n}$ is a new minimal set of generators of $S[n]_{+}$. Thus each $\varphi \in \operatorname{Aut}(S[n])$ induces an element in $\operatorname{Aut}\left(S[n] / S[n]_{+}^{s+1}\right)$ for each $s$ that, improperly, we again denote by $\varphi$. The algebra $S[n] / S[n]_{+}^{s+1}$ is also a finitely generated vector space on $k$; we fix the basis

$$
\mathcal{X}:=\left(x^{\alpha}\right)_{\alpha \in \mathbb{N}^{n},|\alpha| \leq s}
$$

given by the monomials ordered first by increasing degree and then lexicographically. Thus we can identify each element of $\operatorname{Aut}\left(S[n] / S[n]_{+}^{s+1}\right)$ with a suitable square matrix. It follows that we check the dual basis in $P[n]_{\leq s}$, with respect to the perfect pairing $\langle\cdot, \cdot\rangle$,

$$
\mathcal{Y}:=\left(\frac{1}{\alpha !} y^{\alpha}\right)_{\alpha \in \mathbb{N}^{n},|\alpha| \leq s,}
$$

ordered first by increasing degree and then lexicographically.

Since, in our case, $S[n]_{+}^{s+1} \subseteq \operatorname{Ann}(F)$, it follows that finding the automorphism $\varphi \in \operatorname{Aut}(S[n])$ mapping $\operatorname{Ann}(F)$ to $\operatorname{Ann}\left(F_{\text {simple }}\right)$ is equivalent to finding an automorphism $\widehat{\varphi} \in \operatorname{Aut}\left(S[n] / S[n]_{+}^{s+1}\right)$ mapping $\operatorname{Ann}(F) / S[n]_{+}^{s+1}$ to $\operatorname{Ann}\left(F_{\text {simple }}\right) / S[n]_{+}^{s+1}$. 
By duality, each automorphism $\varphi \in \operatorname{Aut}(S[n])$ mapping $\operatorname{Ann}(F)$ to $\operatorname{Ann}\left(F_{\text {simple }}\right)$ corresponds to an isomorphism

$$
\varphi^{*}:\left(S[n] / \operatorname{Ann}\left(F_{\text {simple }}\right)\right)^{*} \longrightarrow(S[n] / \operatorname{Ann}(F))^{*},
$$

which we interpret as an isomorphism $\varphi^{*}: \operatorname{Ann}\left(F_{\text {simple }}\right)^{\perp} \rightarrow \operatorname{Ann}(F)^{\perp}$ of subspaces of $P[n]_{\leq s} \subseteq P[n]$.

As explained in $[7,8]$, the matrix $M\left(\varphi^{*}\right)$ associated to $\varphi^{*}$ with respect to the basis $\mathcal{Y}$ is exactly the transpose of the inverse of the matrix $M(\varphi)$ associated to the morphism $\varphi$ with respect to the basis $\mathcal{X}$.

We are now ready to prove the main result of the paper. It is a structure theorem for 2-stretched algebras.

Theorem 4.3. Let $A$ be a local, Artinian, Gorenstein k-algebra. Then, $A$ is 2-stretched with $n=H_{A}(1), m=H_{A}(2)$ and $s=\operatorname{sdeg}(A)$ if, and only if,

$$
A \cong S[n] / \operatorname{Ann}(F)
$$

where

$$
F:=y_{1}^{s}+F_{3}+\sum_{j=m+1}^{n} y_{j}^{2}, \quad F_{3} \in P[m]_{3},
$$

$x_{1}^{2} \circ F_{3}=0$ and $x_{2} \circ F_{3}, \ldots, x_{m} \circ F_{3}$ are linearly independent.

Proof. In the following we will set

$$
N(h):=\left\{\alpha \in \mathbb{N}^{m}|| \alpha \mid=h, \alpha \neq h e_{1}\right\} .
$$

We first prove the "if" part. Since $F_{3} \in P[\mathrm{~m}]_{3}$, it follows that $\operatorname{tdf}(F)_{q}=\left\langle s ! y_{1}^{q}+q ! x_{1}^{s-q} \circ F_{3}\right\rangle$, for each $3 \leq q \leq s$. Due to equality (2.1), $H_{A}(q)=\operatorname{dim}_{k}\left(\operatorname{tdf}(F)_{q}\right)=1$ in the same range. Clearly $\left\langle s ! y_{1}^{2}+\right.$ $\left.2 x_{1}^{s-2} \circ F_{3}, x_{2} \circ F_{3}, \ldots, x_{m} \circ F_{3}\right\rangle \subseteq \operatorname{tdf}(F)_{2}$. Since $x_{2} \circ F_{3}, \ldots, x_{m} \circ F_{3}$ are linearly independent and do not contain $y_{1}^{2}$, because $x_{1}^{2} \circ F_{3}=0$, it follows that equality holds; thus, again $H_{A}(2)=\operatorname{dim}_{k}\left(\operatorname{tdf}(F)_{2}\right)=m$.

Similarly $\left\langle s ! y_{1}+x_{1}^{s-1} \circ F_{3}, x^{\gamma} \circ F_{3}, y_{m+1}, \ldots, y_{n}\right\rangle_{\gamma \in N(2)} \subseteq \operatorname{tdf}(F)_{1}$. Again we actually have an equality. Indeed the only possible new element in $\operatorname{tdf}(F)_{1}$ could be a linear combination of $s ! y_{1}^{2}+2 x_{1}^{s-2}$ 。 $F_{3}, x_{2} \circ F_{3}, \ldots, x_{m} \circ F_{3}$ when $s=4$. In addition, the condition $x_{1}^{2} \circ F_{3}=0$ 
guarantees that this cannot occur. It follows that

$$
\operatorname{tdf}\left(F_{\geq 3}\right)_{1}=\left\langle s ! y_{1}+2 x_{1}^{s-1} \circ F_{3}, x^{\gamma} \circ F_{3}\right\rangle_{\gamma \in N(2)} .
$$

We conclude that the Hilbert function of $B:=S[m] /\left(F_{\geq 3}\right)$ is $H_{B}=$ $(1, a, m, 1, \ldots, 1)$. Due to formula $(2.3)$ we know that $a \geq m$. Since $F_{\geq 3} \in P[m]$ we necessarily have

$$
\left\langle s ! y_{1}+2 x_{1}^{s-1} \circ F_{3}, x^{\gamma} \circ F_{3}\right\rangle_{\gamma \in N(2)}=\left\langle y_{1}, \ldots, y_{m}\right\rangle,
$$

whence $H_{A}(1)=n$.

Now we prove the "only if" part. Due to Lemma 4.1 we know that

$$
A \cong S[n] / \operatorname{Ann}(F),
$$

where

$$
F:=y_{1}^{s}+F_{3}+F_{2}+\sum_{j=m+1}^{n} y_{j}^{2}, \quad F_{i} \in P[m]_{i},
$$

$x_{1}^{2} \circ F_{3}=x_{1}^{2} \circ F_{2}=0$ and $x_{2} \circ F_{3}, \ldots, x_{m} \circ F_{3}$ are linearly independent. We first examine the case $n=m$. The changes in the case $n>m$ will be listed at the end of the proof.

Imitating the proof of [7, Theorem 3.3], we look for a suitable automorphism $\varphi \in \operatorname{Aut}\left(S[n]_{\leq s}\right)$ defined as

$$
\varphi\left(x_{j}\right)=x_{j}+\sum_{\gamma \in N(2)} b_{\gamma, j} x^{\gamma}
$$

whose dual morphism transforms $\operatorname{Ann}\left(F_{\text {simple }}\right)^{\perp}$ to $\operatorname{Ann}(F)^{\perp}$. The matrix $M(\varphi)$ with respect to $\mathcal{Y}$ is:

$$
B:=\left(\begin{array}{cccccc}
1 & 0 & 0 & 0 & \cdots & 0 \\
0 & I_{n} & 0 & 0 & \cdots & 0 \\
0 & B(2,1) & I_{\left({ }^{2}+1\right)} & 0 & \cdots & 0 \\
0 & 0 & B(3,2) & I_{(n+2)}^{n+2} & \cdots & 0 \\
0 & 0 & B(4,2) & B(4,3) & \cdots & 0 \\
0 & 0 & 0 & B(5,3) & \cdots & 0 \\
\vdots & \vdots & \vdots & \vdots & \ddots & \vdots \\
0 & 0 & 0 & * & \cdots & I_{\left(\begin{array}{c}
n+e-1 \\
e
\end{array}\right)}
\end{array}\right),
$$


where $I_{h}$ is the identity matrix of order $h, B(i, j)$ are matrices of order $\left(\begin{array}{c}n+i-1 \\ i\end{array}\right) \times\left(\begin{array}{c}n+j-1 \\ j\end{array}\right)$ whose entries are forms of degree $i-j$ in the $b_{\gamma, j}$ 's and 0 is a zero matrix of suitable order.

Set

$$
F_{3}=\sum_{\alpha \in N(3)} \frac{s !}{\alpha !} u_{\alpha} y^{\alpha}, \quad F_{2}=\sum_{\beta \in N(2)} \frac{s !}{\beta !} v_{\beta} y^{\beta} .
$$

We have

$$
\sum_{i=1}^{n} u_{2 e_{1}+e_{i}} y_{i}=x_{1}^{2} \circ F_{3}=0, \quad v_{2 e_{1}}=x_{1}^{2} \circ F_{2}=0 .
$$

We denote by $\Delta$ the $n \times\left(\begin{array}{c}n+1 \\ 2\end{array}\right)$ matrix whose $t$ th row is the vector of the coordinates of $x_{t} \circ F_{3}$ with respect to the basis $\mathcal{Y}$. We notice that the condition $x_{1}^{2} \circ F_{3}=0$ implies that the first column of $\Delta$ is zero.

Let $0_{t}$ be the 0 vector of $k^{\oplus t}$, and let $e$ be the first vector of the canonical basis of $k^{\left(\begin{array}{c}n+s-1 \\ s\end{array}\right.}$ ) (thus $e$ is the vector of the components of $y_{1}^{s}$ with respect to $\mathcal{Y}$ ). The component of $F$ and $F_{\text {simple }}$ with respect to the basis $\mathcal{Y}$ of $P[n]_{\leq s}$ are, respectively,

$$
\begin{aligned}
{[F]_{\mathcal{Y}} } & =\left(0,0_{n}, s ! v_{\beta}, s ! u_{\alpha}, 0_{\left(\begin{array}{c}
n+3 \\
4
\end{array}\right)}, \ldots, 0_{\left(\begin{array}{c}
n+s-2 \\
s-1
\end{array}\right)}, e\right)_{\substack{\beta \in \mathbb{N}^{m},|\beta|=2 \\
\alpha \in \mathbb{N},|\alpha|=3}}, \\
{\left[F_{\text {simple }}\right]_{\mathcal{Y}} } & =\left(0,0_{n}, 0_{\left(\begin{array}{c}
n+1 \\
2
\end{array}\right)}, s ! u_{\alpha}, 0_{\left(\begin{array}{c}
n+3 \\
4
\end{array}\right)}, \ldots, 0_{\left(\begin{array}{c}
n+s-2 \\
s-1
\end{array}\right)}, e\right)_{\substack{\beta \in \mathbb{N} m \\
\alpha \in \mathbb{N}^{m},|\beta|=2 \\
\alpha, \mid \alpha=3}}
\end{aligned}
$$

By duality, we have to look for a $\varphi$ such that

$$
\left(u_{\alpha}\right)_{\alpha \in \mathbb{N}^{m},|\alpha|=3} B(3,2)=\left(v_{\beta}\right)_{\beta \in \mathbb{N}^{m},|\beta|=2} .
$$

Notice that the columns of $B(3,2)$ are exactly the coefficients of the forms of degree 3 in the products

$$
\left(x_{j}+\sum_{\gamma \in \mathbb{N}^{m},|\gamma|=2} b_{\gamma, j} x^{\gamma}\right)\left(x_{h}+\sum_{\delta \in \mathbb{N}^{m},|\delta|=2} b_{\delta, h} x^{\delta}\right)
$$

for $j \leq h=1, \ldots, n$. It follows that the entry on the $\alpha$ th row and on the $(j, h)$ th column is

$$
B(3,2)_{\alpha,(j, h)}= \begin{cases}b_{\delta+e_{j}, h}+b_{\gamma+e_{h}, j} & \text { if } \alpha \geq e_{j}+e_{h}, \\ b_{\delta+e_{j}, h} & \text { if } \alpha \geq e_{j}, \alpha \geq e_{h}, \\ 0 & \text { otherwise. }\end{cases}
$$


Thus, the entries of the product $\left(u_{\alpha}\right)_{\alpha \in N(3)} B(3,2)$ are bihomogeneous forms in the $u_{\alpha}$ 's and $b_{\gamma, j}$ 's. Hence, there is a suitable $n\left(\begin{array}{c}n+1 \\ 2\end{array}\right) \times\left(\begin{array}{c}n+1 \\ 2\end{array}\right)$ matrix $U$ whose coefficients depend on the $u_{\alpha}$ 's and such that

$$
\left(u_{\alpha}\right)_{\alpha \in \mathbb{N}^{m},|\alpha|=3} B(3,2)=\left(b_{\gamma, j}\right)_{\substack{\gamma \in \mathbb{N}^{m},|\gamma|=2 \\ j=1, \ldots, n}}^{t} U
$$

(the $b_{\gamma, j}$ 's are ordered first with respect to $\gamma$ and then with respect to $j$ ). Thus, we obtain from equalities (4.2) and (4.3) the system of linear equations:

$$
U^{t}\left(b_{\gamma, j}\right)_{\substack{\gamma \in \mathbb{N}^{m},|\gamma|=2 \\ j=1, \ldots, n}}={ }^{t}\left(v_{\beta}\right)_{\beta \in \mathbb{N}^{m},|\beta|=2}
$$

in the variables $b_{\gamma, j}$ 's. We recall that, in [7, Proof of Theorem 3.3], it is proved that $U$ is a lower triangular block matrix

$$
U=\left(\begin{array}{cccccc}
U(1) & * & * & \cdots & * & * \\
0 & U(2) & * & \cdots & * & * \\
0 & 0 & U(3) & \cdots & * & * \\
\vdots & \vdots & \vdots & \ddots & \vdots & \vdots \\
0 & 0 & 0 & \cdots & U(n-1) & * \\
0 & 0 & 0 & \cdots & 0 & U(n)
\end{array}\right)
$$

where $U(h)$ is a $(n-h+1) \times\left(\begin{array}{c}n+1 \\ 2\end{array}\right)$ matrix whose first row is twice the $h$ th row of the matrix $\Delta$ (previously defined as the matrix of the partial derivatives of $\left.F_{3}\right)$ and the $t$ th row is exactly the $(h+t-1)$ th row of $\Delta, t=2, \ldots, n-h+1$.

Due to the independence of the derivatives $x_{2} \circ F_{3}, \ldots, x_{m} \circ F_{3}$ it thus follows that the rank of the submatrix obtained by erasing the first row of $U$ is maximal. Moreover, the constant term of the first equation is $v_{2 e_{1}}$ which is zero, because $x_{1}^{2} \circ F_{2}=0$. It follows that the existence of a solution of the system (4.4) with $b_{\gamma, 1}=0, \gamma \in \mathbb{N}^{m}$ and $|\gamma|=2$.

In order to extend the above proof to the case $n>m$ it suffices to change the ordering on $\mathcal{X}$ and, consequently, on $\mathcal{Y}$. In this case we fix an order on $\mathcal{X}$ by first taking all the monomials in $x_{1}, \ldots, x_{m}$ (ordered first by degree and then lexicographically), and then all the remaining monomials in any order. Thus,

$$
M(\varphi)=\left(\begin{array}{cc}
B & 0 \\
* & B^{\prime}
\end{array}\right)
$$


where $B$ is as in (4.1) and $B^{\prime}$ is a suitable matrix whose entries depend on the $b_{\gamma, j}$ 's such that $\gamma \geq e_{j}$ for some $j \geq m+1$. We can thus repeat the above arguments, obtaining a system of the form:

$$
\left(\begin{array}{cc}
U & * \\
0 & U^{\prime}
\end{array}\right)^{t}\left(b_{\gamma, j}\right)_{\substack{\gamma \in \mathbb{N}^{m},|\gamma|=2 \\
j=1, \ldots, n}}={ }^{t}\left(v_{\beta}\right)_{\beta \in \mathbb{N}^{m},|\beta|=2},
$$

where $U$ is as above and the entries of $U^{\prime}$ depend on $u_{\alpha}$ such that $\alpha \geq e_{j}$ for some $j \geq m+1$. On the one hand, due to Lemma 4.1 we know that such $u_{\alpha}$ are all zero, i.e., $U^{\prime}$ is the zero matrix. On the other hand, again by Lemma 4.1 we know that $v_{\beta}=0$, for $\beta \geq e_{j}$, when $j \geq m+1$. We deduce that such a system again has solutions, and, in particular, one of its solutions satisfies $b_{\gamma, j}=0, \gamma \in \mathbb{N}^{m},|\gamma|=2$, $j=1, m+1, \ldots, n$.

5. Obstructedness of a class of algebras. In this section, we make use of the above structure theorem in order to deal with the obstructedness of the points in $\mathcal{H i l b} b_{11}^{G}\left(\mathbb{P}_{k}^{N}\right)$ corresponding to schemes $X \cong \operatorname{spec}(A)$ where $A$ is a local, Artin, Gorenstein $k$-algebra with Hilbert function $H_{A}=(1,4,4,1,1)$. Thus, $A \cong S[4] / J$, where $J$ contains

$$
x^{\beta}, x^{\alpha}, \quad \beta, \alpha \in \mathbb{N}^{4},|\beta|=4, \beta \neq 4 e_{1},|\alpha|=5 .
$$

It follows that $S[4]_{+}^{5} \subseteq J$; hence, there is a natural isomorphism

$$
\frac{S[4]}{J} \cong \frac{k\left[x_{1}, x_{2}, x_{3}, x_{4}\right]}{J \cap k\left[x_{1}, x_{2}, x_{3}, x_{4}\right]},
$$

inducing a natural epimorphism $k\left[x_{1}, x_{2}, x_{3}, x_{4}\right] \rightarrow A \cong S[4] / J$, i.e., an embedding $X \subseteq \mathbb{A}_{k}^{4} \subseteq \mathbb{P}_{k}^{4}$.

In [5], we proved the irreducibility of $\mathcal{H i l b} b_{11}^{G}\left(\mathbb{P}_{k}^{n}\right)$ by studying the locus of singular $X$ such that $X \cong \operatorname{spec}(A)$, where $A$ is local with $H_{A} \neq(1,4,4,1,1)$. Thus, a point $X \in \mathcal{H} i l b_{11}\left(\mathbb{P}_{k}^{n}\right)$ is singular, i.e., the corresponding scheme is obstructed, if the dimension of the tangent space at $X$ to $\mathcal{H} i l b_{11}\left(\mathbb{P}_{k}^{n}\right)$ is greater than $\operatorname{dim}\left(\mathcal{H i l b} b_{11}^{G}\left(\mathbb{P}_{k}^{n}\right)\right)$.

Obstructedness depends only on the intrinsic structure of $X$ (see [3] and the references therein), hence only on $A$. Thus, we can restrict our attention to the aforementioned embedding in $\mathbb{P}_{k}^{4}$, and we simply speak about the obstructedness of the algebra $A$. 
Recall that the tangent space to $\mathcal{H} i l b_{11}^{G}\left(\mathbb{P}_{k}^{4}\right)$ at $X$ is canonically identified with $H^{0}(X, \mathcal{N})$, global sections of the normal sheaf $\mathcal{N}$ of $X:=\operatorname{spec}(S[4] / J) \subseteq \mathbb{A}_{k}^{4}$. Thus, $X$ is unobstructed if and only if

$$
N_{S[4] / J}:=h^{0}(X, \mathcal{N})=\operatorname{dim}\left(\mathcal{H i l b} b_{11}^{G}\left(\mathbb{P}_{k}^{4}\right)\right)=44 .
$$

In [3], we pointed out that $N_{S[4] / J}=\operatorname{dim}_{k}\left(S[4] / J^{2}\right)-11$.

Due to Theorem 4.3, we can assume $J=\operatorname{Ann}(F)$, where $F=y_{1}^{4}+F_{3}$, $F_{3} \in P[4], x_{1}^{2} \circ F_{3}=0$ and $x_{2} \circ F_{3}, x_{3} \circ F_{3}, x_{4} \circ F_{3}$ are linearly independent. So,

$$
F=y_{1}^{4}+y_{1} Q+H,
$$

where $Q \in k\left[y_{2}, y_{3}, y_{4}\right]_{2}$ and $H \in k\left[y_{2}, y_{3}, y_{4}\right]_{3}$. Either $H=0$ or, up to a suitable linear transformation of the variables $y_{2}, y_{3}, y_{4}$, is one of the following:

$$
\begin{gathered}
y_{2}^{3}+y_{3}^{3}+y_{4}^{3}+t y_{2} y_{3} y_{4}, \quad y_{2}^{3}+y_{3}^{3}+y_{2} y_{3} y_{4}, \\
y_{2}^{3}+y_{2} y_{3} y_{4}, \quad y_{2} y_{3} y_{4}, \\
y_{2}^{3}+y_{3}^{2} y_{4}, \\
y_{2}^{2} y_{3}+y_{3}^{2} y_{4}, \quad y_{3}^{2} y_{4}-y_{3} y_{4}^{2}, \quad y_{3} y_{4}^{2}, \quad y_{4}^{3} .
\end{gathered}
$$

We will compute $\operatorname{dim}_{k}\left(S[4] / J^{2}\right)$ case by case.

Before starting with the description in the different cases we give descriptions of the methods used to perform the computations.

Due to relations (5.1), we know that $S[4]_{+}^{10} \subseteq J^{2}$; thus,

$$
\frac{S[4]}{J^{2}} \cong \frac{k\left[x_{1}, x_{2}, x_{3}, x_{4}\right]}{J^{2} \cap k\left[x_{1}, x_{2}, x_{3}, x_{4}\right]},
$$

too. In particular, when we perform computations, we can always work in the polynomial ring $k\left[x_{1}, x_{2}, x_{3}, x_{4}\right]$ instead of $S[4]$. For this reason, we can make use of the computer algebra software Singular [6] for all the computations in $S[4]$.

Moreover, $J$ is never homogeneous. We computed the initial ideals in $(J)$ and in $\left(J^{2}\right)$ with respect to the product term order for which

(i) $x_{4}>x_{3}>x_{2}>x_{1}$;

(ii) the graded reverse lexicographic order on $x_{4}, x_{3}, x_{2}$;

(iii) the lexicographic order on $x_{1}$. 
Hence, in order to compare $x_{4}^{a_{4}} x_{3}^{a_{3}} x_{2}^{a_{2}} x_{1}^{a_{1}}$ and $x_{4}^{b_{4}} x_{3}^{b_{3}} x_{2}^{b_{2}} x_{1}^{b_{1}}$, we first compare $x_{4}^{a_{4}} x_{3}^{a_{3}} x_{2}^{a_{2}}$ and $x_{4}^{b_{4}} x_{3}^{b_{3}} x_{2}^{b_{2}}$ with respect to degrevlex, and, if they are equal, we compare $x_{1}^{a_{1}}$ and $x_{1}^{b_{1}}$ with respect to lex.

For such a choice, the Hilbert function of $S[4] / \operatorname{in}(J)$ is $(1,4,4,1,1)$, while the Hilbert function of $S[4] / \operatorname{in}\left(J^{2}\right)$ has to be computed case by case, as $Q$ and $H$ vary.

We start by examining the case $H=0$.

Proposition 5.1. Let $A:=S[4] / \operatorname{Ann}\left(y_{1}^{4}+y_{1} Q\right)$ with $Q \in k\left[y_{2}, y_{3}, y_{4}\right]_{2}$ and $H_{A}=(1,4,4,1,1)$. Then $N_{A}=49$; hence, $A$ is obstructed.

Proof. We know that $x_{i} \circ F_{3}, i=2,3,4$, are linearly independent because of Theorem 4.3. It follows that, up to a linear change of the variables $y_{2}, y_{3}, y_{4}$, we can assume $Q=y_{2}^{2}+y_{3}^{2}+y_{4}^{2}$. In this case, $J=\operatorname{Ann}(F)$ is generated by

$$
\begin{gathered}
x_{4}^{2}-x_{2}^{2}, \quad x_{3}^{2}-x_{2}^{2}, \quad x_{4} x_{3}, \quad x_{4} x_{2}, \\
x_{3} x_{2}, \quad 12 x_{4}^{2}-x_{1}^{3}, \quad x_{2} x_{1}^{2}, \quad x_{3} x_{1}^{2}, \quad x_{4} x_{1}^{2} .
\end{gathered}
$$

Using Singular, we obtain $H_{S[4] / J^{2}}=(1,4,10,20,20,4,1)$; thus, $N_{A}=49>44$.

Remark 5.2. The hypothesis $H_{A}=(1,4,4,1,1)$ in Proposition 5.1 is equivalent to $\operatorname{rk}(M)=3$, where $M$ is the symmetric matrix associated to the quadratic form $Q$.

Now we turn our attention to the case $H \neq 0$. It follows that $H$ is one of the polynomials indicated in the list (5.2). We outline the strategy for the computations.

Once $H \neq 0$ is fixed, for each $b:=\left(b_{0}, \ldots, b_{5}\right) \in \mathbb{A}_{k}^{6}$, we consider the quadratic form:

$$
Q_{b}:=b_{0} y_{2}^{2}+2 b_{1} y_{2} y_{3}+b_{2} y_{3}^{2}+2 b_{3} y_{2} y_{4}+2 b_{4} y_{3} y_{4}+b_{5} y_{4}^{2}
$$

The associated symmetric matrix is the matrix $M_{b}$ defined in the introduction.

We define $F^{H, b}:=y_{1}^{4}+y_{1} Q_{b}+H$ and $A^{H, b}:=S[4] / \operatorname{Ann}\left(F^{H, b}\right)$. Hence, $\operatorname{Ann}\left(F^{H, b}\right)$ depends on $x_{1}, \ldots, x_{4}$ and $b_{0}, \ldots, b_{5}$. Due to The- 
orem 4.3 and [11, page 415, Corollary], we have a flat family whose base is the open non-empty subset

$$
\begin{aligned}
B_{H}:=\left\{b \in \mathbb{A}_{k}^{6} \mid \text { where } x_{i} \circ\left(y_{1} Q_{b}+H\right),\right. \\
\\
\quad i=2,3,4, \text { are linearly independent }\} .
\end{aligned}
$$

Trivially, we must compute the Hilbert function of $\operatorname{Ann}\left(F^{H, b}\right)^{2}$ as a function of $b$.

The following result helps us to simplify the computations in several cases.

Lemma 5.3. Let $H \in k\left[y_{2}, y_{3}, y_{4}\right]_{3}$ be fixed. If $0 \in B_{H}$ and $A^{H, 0}$ is unobstructed, then $B_{H}=\mathbb{A}_{k}^{6}$ and, for every $b \in \mathbb{A}_{k}^{6}$, we have that $A^{H, b}$ is unobstructed.

Proof. Fix $b$ and $H$. Theorem 4.3 implies that $H_{A^{H, 0}}=(1,4,4,1,1)$ because $0 \in B_{H}$. In particular, $x_{i} \circ F_{3}^{H, 0}=x_{i} \circ H, i=2,3,4$, are linearly independent; thus, the same is true for $x_{i} \circ F_{3}^{H, t b}, i=2,3,4$, without restrictions on $t \in k$ and $b \in \mathbb{A}_{k}^{6}$, because $y_{1}$ does not appear in $H$. In particular, $B_{H}=\mathbb{A}_{k}^{6}$.

Again, Theorem 4.3 implies that the Hilbert function of $A^{H, t b}$ is $(1,4,4,1,1)$; thus, we have a flat family $([\mathbf{1 1}$, page 415 , Corollary $])$ of deformations of $A^{H, 0}$ with base $\mathbb{A}_{k}^{1}$. If $t \neq 0$, the automorphism of $P[4]$ defined by

$$
\left(y_{1}, y_{2}, y_{3}, y_{4}\right) \longmapsto\left(t^{3} y_{1}, t^{4} y_{2}, t^{4} y_{3}, t^{4} y_{4}\right)
$$

shows that all the other deformations are isomorphic to $A^{H, b}$. Since $A^{H, 0}$ is unobstructed, it follows that the general one, i.e., $A^{H, b}$, is unobstructed too.

As an immediate application of Lemma 5.3 we obtain the following general result.

Proposition 5.4. Let $H$ be either $y_{2}^{3}+y_{3}^{3}+y_{4}^{3}+t y_{2} y_{3} y_{4}$ with $t\left(t^{3}-\right.$ 216) $\neq 0$, or $y_{2}^{3}+y_{3}^{3}+y_{2} y_{3} y_{4}, y_{2}^{3}+y_{2} y_{3} y_{4}$, or $y_{2} y_{3} y_{4}$. Then $B_{H}=\mathbb{A}_{k}^{6}$, and $A^{H, b}$ is unobstructed.

Proof. In all cases but the first one, we apply the same strategy as in Proposition 5.1. We compute the generators of the ap- 
olar ideal $J$ for $Q_{b}=0$, and we compute the Hilbert function of $S[4] / J^{2}$ by using Singular that turns out to be equal to $H_{S[4] / J^{2}}=$ $(1,4,10,20,14,4,1,1)$ in all the cases. Hence, $0 \in B_{H}$, and $A^{H, 0}$ is unobstructed. The result then follows from Lemma 5.3.

Now we consider the first case. We will write $H_{t}$ instead of $H$. As a first stage, we look for $t$ such that $0 \in \mathcal{U}_{t}$, where $\mathcal{U}_{t} \subseteq \mathbb{A}_{k}^{6}$ is the open subset of $b$ 's such that $A^{H_{t}, b}$ is unobstructed. For such values of $t$, we have $B_{H_{t}}=\mathbb{A}_{k}^{6}$ again by Lemma 5.3 .

The ideal $J_{t}:=\operatorname{Ann}\left(F^{H_{t}, 0}\right)$ is non-minimally generated by

$$
\begin{gathered}
x_{1} x_{2}, \quad x_{1} x_{3}, \quad x_{1} x_{4}, \quad t x_{2}^{2}-6 x_{3} x_{4}, \quad t x_{3}^{2}-6 x_{2} x_{4}, \\
t x_{4}^{2}-6 x_{2} x_{3}, \quad x_{1}^{2} x_{2}, \quad x_{1} x_{2}^{2}, \quad x_{1}^{2} x_{3}, \quad x_{1} x_{2} x_{3}, \\
x_{2}^{2} x_{3}, \quad x_{1} x_{3}^{2}, \quad x_{2} x_{3}^{2}, \quad x_{1}^{2} x_{4}, \quad x_{1} x_{2} x_{4}, \quad x_{2}^{2} x_{4}, \\
x_{1} x_{3} x_{4}, \quad x_{3}^{2} x_{4}, \quad x_{1} x_{4}^{2}, \quad x_{2} x_{4}^{2}, \quad x_{3} x_{4}^{2}, \quad 4 x_{2}^{3}-x_{1}^{4}, \\
4 x_{3}^{3}-x_{1}^{4}, \quad 24 x_{2} x_{3} x_{4}-t x_{1}^{4}, \quad 4 x_{4}^{3}-x_{1}^{4} .
\end{gathered}
$$

By computing a Gröbner basis of $J_{t}$, we get that, if $t \neq 0$, the initial ideal of $J_{t}$ does not depend on $t$, and the Hilbert function $H_{S[4] / J}=(1,4,4,1,1)$, as expected.

The coefficients of the initial terms of the polynomials of a Gröbner basis of $J_{t}^{2}$ are either non-zero constants, multiples of $t$, or multiples of $t^{3}-216$, e.g., $t x_{4}^{5}-6 x_{2}^{4} x_{3},\left(t^{3}-216\right) x_{2}^{4} x_{4}$. Thus, if $t\left(t^{3}-216\right) \neq 0$, the Hilbert function $H_{S[4] / J_{t}^{2}}$ does not depend on $t$ and is the same one computed in previous cases. So the proof of the statement follows by using the same previous arguments also in the first case.

Remark 5.5. The form $y_{2}^{3}+y_{3}^{3}+y_{4}^{3}+t y_{2} y_{3} y_{4}$ is the sum of the cubes of three linearly independent linear forms, i.e., it represents a Fermat cubic in the projective plane, if, and only if, $t\left(t^{3}-216\right)=0$.

In the remaining cases of list (5.2) plus the Fermat cubic, the above argument does not work. Anyhow, as will be evident from the computations below, the generators of $\operatorname{Ann}\left(F^{H, b}\right)$, hence of $\operatorname{Ann}\left(F^{H, b}\right)^{2}$, in $k[b] \otimes_{k} S[4]$, have homogeneous coefficients in $k[b]$, and this property still holds when we compute Gröbner bases of either $\operatorname{Ann}\left(F^{H, b}\right)$ or $\operatorname{Ann}\left(F^{H, b}\right)^{2}$. 
Now, we focus on $\operatorname{Ann}\left(F^{H, b}\right)^{2}$. The Hilbert function of the quotient $S[4] / \operatorname{Ann}\left(F^{H, b}\right)^{2}$ will be constant on an open subset of $B_{H}$. If the Hilbert function of $S[4] / \operatorname{Ann}\left(F^{H, b}\right)^{2}$ changes for suitable $b$, then $\operatorname{in}\left(\operatorname{Ann}\left(F^{H, b}\right)^{2}\right)$ must change as well. Of course, the converse does not hold. The initial ideal in $\left(\operatorname{Ann}\left(F^{H, b}\right)^{2}\right)$ could change but the Hilbert function of $S[4] / \operatorname{Ann}\left(F^{H, b}\right)^{2}$ stays the same. However, we compute a Gröbner basis of $\operatorname{Ann}\left(F^{H, b}\right)^{2}$ with respect to the term order described above, and we compute, for each monomial of $\operatorname{in}\left(\operatorname{Ann}\left(F^{H, b}\right)^{2}\right)$, the homogeneous ideals in $k[b]$ spanned by its coefficients. We compute the prime ideals $r_{*}$ associated to such ideals, giving us a set of level 1 conditions that force the initial ideal of $\operatorname{Ann}\left(F^{H, b}\right)^{2}$ to change. Of course, we can restrict to the associated prime ideals because we study set-theoretically the family $S[4] / \operatorname{Ann}\left(F^{H, b}\right) \rightarrow B_{H}$. By computing the Gröbner basis of $r_{*}+\operatorname{Ann}\left(F^{H, b}\right)^{2}$, we get a new initial ideal that we study exactly as before, obtaining a new set of level 2 prime ideals. Continuing with this strategy, we construct a tree that we analyze leafby-leaf from the point of view of the Hilbert function.

Before listing the results, we explain how we perform the computations.

Computational strategy. The Gröbner bases computations will be performed in the ring $k\left[x_{4}, \ldots, x_{1}, b_{0}, \ldots, b_{5}\right]$, up to choosing a product term ordering, with 3 blocks of variables: $x_{4}>x_{3}>x_{2}, x_{1}, b_{0}>\cdots>$ $b_{5}$, and degrevlex orders the monomials in the first and last block, while lex orders the monomials in the second block.

The steps of the computations are as follows.

(1) To compute the Hilbert function of $\operatorname{Ann}\left(F^{H, b}\right)^{2}$ over the general element of the variety $V$ defined by a prime ideal $r \subset k[b]$, we proceed in the following way. First, we compute a Gröbner basis of $r b=r+\operatorname{Ann}\left(F^{H, b}\right)^{2}$, then the initial ideal in $(r b)$, generated by the initial terms of the polynomial in the computed Gröbner basis, then erase from in $(r b)$ the monomials contained in $k[b]$, and finally we reduce the resulting monomial ideal by setting $b_{i}=1$ for each $i$. As a last step, we compute the Hilbert function of the ideal we get.

(2) To compute the ideal spanned by the coefficients of a particular initial monomial $M \in k\left[x_{1}, \ldots, x_{4}\right]$, we select the polynomials in the Gröbner basis having initial monomial $M M^{\prime}$ with 
$M^{\prime} \in k\left[b_{0}, \ldots, b_{5}\right]$. Then we compute the remainder of every such polynomial modulo $M$ and the difference $D$ between the polynomial and its remainder. $D$ has $M$ as a factor, and we call $d$ the quotient. The computed $d$ 's are the generators of the ideal spanned by the coefficients. The prime ideals associated to such an ideal are computed by using the package Primdec in Singular, when it produces the result (this happened except for a few cases in which we had to make the computation by hand with ad hoc techniques, because the size of the ideal was too big for Singular to compute the result in a reasonable time).

In next proposition, we deal with the cases $H \neq 0$ not covered by Proposition 5.4.

Proposition 5.6. Let $H=y_{2}^{3}+y_{3}^{3}+y_{4}^{3}$. Then $B_{H}=\mathbb{A}_{k}^{6}$ and $A^{H, b}$ is obstructed if, and only if, $b \in V\left(b_{1}, b_{3}, b_{4}\right) \subseteq \mathbb{A}_{k}^{6}$.

Let $H=y_{2}^{3}+y_{3}^{2} y_{4}$. Then $B_{H}=\mathbb{A}_{k}^{6}$ and $A^{H, b}$ is obstructed if, and only if, $b \in V\left(b_{1}, b_{3}, b_{5}\right) \subseteq \mathbb{A}_{k}^{6}$.

Let $H=y_{2}^{2} y_{3}+y_{3}^{2} y_{4}$. Then $B_{H}=\mathbb{A}_{k}^{6}$ and $A^{H, b}$ is obstructed if, and only if, $b \in V\left(b_{0}-b_{4}, b_{3}, b_{5}\right) \subseteq \mathbb{A}_{k}^{6}$.

Let $H=y_{3}^{2} y_{4}-y_{3}^{2} y_{4}$. Then $B_{H}=\mathbb{A}_{k}^{6} \backslash V\left(b_{0}, b_{1}, b_{3}\right)$ and $A^{H, b}$ is obstructed if, and only if, $b \in V\left(-b_{1}^{2}+b_{0} b_{2}-b_{1} b_{3}-b_{3}^{2}+b_{0} b_{4}+b_{0} b_{5}\right) \subseteq \mathbb{A}_{k}^{6}$.

Let $H=y_{3} y_{4}^{2}$. Then $B_{H}=\mathbb{A}_{k}^{6} \backslash V\left(b_{0}, b_{1}, b_{3}\right)$ and $A^{H, b}$ is obstructed if, and only if, $b \in V\left(b_{1}^{2}-b_{0} b_{2}\right) \subseteq \mathbb{A}_{k}^{6}$.

Let $H=y_{4}^{3}$. Then $B_{H}=\left\{b \in \mathbb{A}_{k}^{6} \mid \operatorname{rk}\left(M_{b}\right) \geq 2\right\}$ and $A^{H, b}$ is obstructed for each $b \in B_{H}$.

In all the aforementioned cases, if $A^{H, b}$ is obstructed, then $N_{A^{H, b}}=$ 49.

Proof. In each of the above cases we indicate a non-minimal set of generators of the ideal $\operatorname{Ann}\left(F^{H, b}\right)$ and the locus in $B_{H}$ corresponding to obstructed ideals. Only in the first case of the list we report also the tree and the Hilbert function of the general element of each subset of $B_{H}$ where the initial ideal changes.

Let $H=y_{2}^{3}+y_{3}^{3}+y_{4}^{3}$. It is immediate to check that $B_{H}=\mathbb{A}_{k}^{6}$. Again we will denote by $\mathcal{U}$ the open and non-empty subset of $b \in \mathbb{A}_{k}^{6}$ 
such that $A^{H, b}$ is unobstructed. Since $H$ is fixed throughout the whole proof we will simply write $J_{b}$ instead of $\operatorname{Ann}\left(F^{H, b}\right)$. $J_{b}$ is non-minimally generated by both the monomials in list (5.1) and

$$
\begin{gathered}
3 x_{2} x_{1}-b_{0} p_{1}-b_{1} p_{2}-b_{3} p_{3}, \quad 3 x_{3} x_{1}-b_{1} p_{1}-b_{2} p_{2}-b_{4} p_{3}, \\
12 x_{3} x_{2}-b_{1} x_{1}^{3}, \quad 3 x_{4} x_{1}-b_{3} p_{1}-b_{4} p_{2}-b_{5} p_{3}, \\
12 x_{4} x_{2}-b_{3} x_{1}^{3}, \quad 12 x_{4} x_{3}-b_{4} x_{1}^{3}, \quad x_{2} x_{1}^{2}, \quad 12 x_{2}^{2} x_{1}-b_{0} x_{1}^{4}, \\
4 x_{2}^{3}-x_{1}^{4}, \quad x_{3} x_{1}^{2}, \quad 12 x_{3} x_{2} x_{1}-b_{1} x_{1}^{4}, \quad x_{3} x_{2}^{2}, \\
12 x_{3}^{2} x_{1}-b_{2} x_{1}^{4}, \quad x_{3}^{2} x_{2}, \quad 4 x_{3}^{3}-x_{1}^{4}, \quad x_{4} x_{1}^{2}, \\
12 x_{4} x_{2} x_{1}-b_{3} x_{1}^{4}, \quad x_{4} x_{2}^{2}, \quad 12 x_{4} x_{3} x_{1}-b_{4} x_{1}^{4}, \quad x_{4} x_{3} x_{2}, \quad x_{4} x_{3}^{2}, \\
12 x_{4}^{2} x_{1}-b_{5} x_{1}^{4}, \quad x_{4}^{2} x_{2}, \quad x_{4}^{2} x_{3}, \quad 4 x_{4}^{3}-x_{1}^{4},
\end{gathered}
$$

where

$$
p_{1}=x_{2}^{2}-b_{0} x_{1}^{3} / 12, \quad p_{2}=x_{3}^{2}-b_{2} x_{1}^{3} / 12, \quad p_{3}=x_{4}^{2}-b_{5} x_{1}^{3} / 12 .
$$

We know $0 \notin \mathcal{U}$, because $N_{S[4] / J_{0}}=49$. We have that in $\left(J_{b}^{2}\right)$ could change only if $b$ is in the variety $V\left(r_{*}\right) \subseteq \mathbb{A}_{k}^{6}$ where $r_{*}$ is one of the following ideals, computed according to point (2) of the computational strategy, ordered first by decreasing codimension and then by increasing degree:

$$
\begin{aligned}
& \text { - } r_{1}=\left(b_{1}, b_{3}, b_{4}\right) ; \\
& \text { - } r_{2}=\left(b_{3}, b_{4}, b_{5}\right) ; \\
& \text { - } r_{3}=\left(b_{0}, b_{1}, b_{3}\right) ; \\
& \text { - } r_{4}=\left(b_{1}, b_{2}, b_{4}\right) ; \\
& \text { - } r_{5}=\left(-b_{4}^{2}+b_{2} b_{5},-b_{3} b_{4}+b_{1} b_{5},-b_{2} b_{3}+b_{1} b_{4},-b_{3}^{2}+b_{0} b_{5},-b_{1} b_{3}+\right. \\
&\left.\quad b_{0} b_{4},-b_{1}^{2}+b_{0} b_{2}\right) ; \\
& \text { - } r_{6}=\left(b_{1}, b_{3}\right) ; \\
& \text { - } r_{7}=\left(b_{1}, b_{4}\right) ; \\
& \text { - } r_{8}=\left(b_{3}, b_{4}\right) ; \\
& \text { - } r_{9}=\left(-b_{2} b_{3}+b_{1} b_{4},-b_{1} b_{3}+b_{0} b_{4},-b_{1}^{2}+b_{0} b_{2}\right) ; \\
& \text { - } r_{10}=\left(-b_{4}^{2}+b_{2} b_{5},-b_{3} b_{4}+b_{1} b_{5},-b_{2} b_{3}+b_{1} b_{4}\right) ; \\
& \text { - } r_{11}=\left(-b_{3} b_{4}+b_{1} b_{5},-b_{3}^{2}+b_{0} b_{5},-b_{1} b_{3}+b_{0} b_{4}\right) ; \\
& \text { - } r_{12}=\left(b_{3}\right) ; \\
& \text { - } r_{13}=\left(b_{1}\right) ; \\
& \text { - } r_{15}=\left(-b_{1}^{2}+b_{0} b_{3}\right) ;
\end{aligned}
$$


- $r_{16}=\left(-b_{2} b_{3}^{2}+2 b_{1} b_{3} b_{4}-b_{0} b_{4}^{2}-b_{1}^{2} b_{5}+b_{0} b_{2} b_{5}\right)$;

- $r_{17}=\left(b_{1} b_{2}^{2} b_{3}^{2}-2 b_{1}^{2} b_{2} b_{3} b_{4}+b_{1}^{3} b_{4}^{2}-b_{3}^{3} b_{4}^{2}+2 b_{1} b_{3}^{2} b_{4} b_{5}-b_{1}^{2} b_{3} b_{5}^{2}\right)$.

These ideals are the first level conditions in the tree we are constructing. Now, we impose the conditions one at a time, i.e., we compute a Gröbner basis of $r_{*}+J_{b}^{2}$, i.e., $J_{b}^{2}$ restricted to $r_{*}$, and we analyze its initial ideal.

We consider the ideals $r_{1}, \ldots, r_{17}$ in the given order.

Let us first look at $r_{1}$. For a general $b \in V\left(r_{1}\right)$, we have that $H_{S[4] / J_{b}^{2}}=(1,4,10,20,16,7,1,1)$ (see point (1) of the computational strategy) and so $N_{S[4] / J_{b}}=49$. Hence, $A^{H, b}$ is obstructed for a general $b \in V\left(r_{1}\right)$. Since $V\left(r_{1}\right)$ is irreducible, it follows that $V\left(r_{1}\right) \cap \mathcal{U}=\emptyset$ due to the semicontinuity of the Hilbert function of $S[4] / J_{b}^{2}$. The following computations will prove that actually $V\left(r_{1}\right)=\mathbb{A}_{k}^{6} \backslash \mathcal{U}$.

Consider $r_{2}$. For a general $b \in V\left(r_{2}\right), A^{H, b}$ is unobstructed because $H_{S[4] / J_{b}^{2}}=(1,4,10,20,14,5,1)$, (see point (1) of the computational strategy) and so $N_{S[4] / J_{b}}=44$.

As we always refer to point (1) of the computational strategy when we compute the Hilbert function of $S[4] / J_{b}^{2}$ for a general $b$, we omit the reference from now on.

The conditions that force the initial ideal to change obviously contain $r_{2}$. They are:

- $r_{2,1}=\left(b_{1}, b_{3}, b_{4}, b_{5}\right)$;

- $r_{2,2}=\left(b_{0}, b_{1}, b_{3}, b_{4}, b_{5}\right)$;

- $r_{2,3}=\left(-b_{1}^{2}+b_{0} b_{2}, b_{3}, b_{4}, b_{5}\right)$;

- $r_{2,4}=\left(b_{1}, b_{2}, b_{3}, b_{4}, b_{5}\right)$.

Notice that $r_{2,2}, r_{2,4} \supset r_{2,1} \supset r_{1}$. From the analysis of $r_{1}$, it follows that $A^{H, b}$ is obstructed for every $b \in V\left(r_{2,2}\right) \cup V\left(r_{2,4}\right) \cup V\left(r_{2,1}\right)=V\left(r_{2,1}\right)$. By using Singular, we check that $N_{S[4] / J_{b}}=49$. On the other hand, if $b \in V\left(r_{2,3}\right) \backslash V\left(r_{2,1}\right)$, then $b \in \mathcal{U}$ because $H_{S[4] / J_{b}^{2}}$ is equal to the one for $b$ general in $V\left(r_{2}\right)$. The conditions that force the initial ideal of $r_{2,3}+J_{b}^{2}$ to change are $r_{2,2}, r_{2,4}$ and $r_{0}=\left(b_{0}, \ldots, b_{5}\right)$. They were studied before, and so the analysis of this case is finished.

Further, we consider $r_{3}$. We first notice that a general $b \in V\left(r_{3}\right)$ corresponds to an unobstructed scheme $A^{H, b}$. There are many conditions that force the initial ideal to change, but many of them were 
already examined above, e.g., $r_{2,2}$. An easy and careful check of such conditions yields that the only totally new ones are

- $r_{3,1}=\left(b_{0}, b_{1}, b_{3}, b_{4}\right)$;

- $r_{3,2}=\left(b_{0}, b_{1}, b_{2}, b_{3}, b_{4}\right)$;

- $r_{3,3}=\left(-b_{4}^{2}+b_{2} b_{5}, b_{3}, b_{1}, b_{0}\right)$.

Notice that $V\left(r_{3,1}\right), V\left(r_{3,2}\right) \subseteq V\left(r_{1}\right) \subseteq \mathbb{A}_{k}^{6} \backslash \mathcal{U}$. Moreover, $A^{H, b}$ is unobstructed for a general $b \in V\left(r_{3,3}\right)$. As the further conditions which force the initial ideal to change are contained in $V\left(r_{1}\right)$, we conclude that

$$
V\left(r_{3,3}\right) \backslash V\left(r_{1}\right) \subset \mathcal{U} .
$$

Also for $V\left(r_{4}\right)$, the general $b \in V\left(r_{4}\right)$ is associated to an unobstructed scheme $A^{H, b}$. The conditions that force the initial ideal to change are:

- $r_{4,1}=\left(b_{1}, b_{2}, b_{3}, b_{4}\right)$;

- $r_{4,2}=\left(-b_{3}^{2}+b_{0} b_{5}, b_{4}, b_{2}, b_{1}\right)$.

The first one defines a subvariety of $V\left(r_{1}\right)$, and so we do not study it further. If $b \in V\left(r_{4,2}\right) \backslash V\left(r_{1}\right)$, then $b \in \mathcal{U}$ for the same argument used to study $V\left(r_{3,3}\right) \backslash V\left(r_{1}\right)$. A similar argument holds for $V\left(r_{5}\right) \backslash V\left(r_{1}\right)$, too.

Now, we start studying the codimension 2 ideals that appear in the initial list. The first we consider is $r_{6}$. Again, the general $b \in V\left(r_{5}\right)$ is in $\mathcal{U}$, and the only new condition that forces the initial ideal to change is

- $r_{6,1}=\left(-b_{4}^{2}+b_{2} b_{5}, b_{3}, b_{1}\right)$.

By using the same technique, we get that $b \in \mathcal{U}$ if, and only if, $b \notin V\left(r_{1}\right)$. Thus, there is no level 3 condition to analyze. A similar argument holds for $r_{7}$ and $r_{8}$. In this case, the only new conditions are:

- $r_{7,1}=\left(-b_{3}^{2}+b_{0} b_{5}, b_{4}, b_{1}\right)$;

- $r_{8,1}=\left(-b_{1}^{2}+b_{0} b_{2}, b_{4}, b_{3}\right)$.

$b \in \mathcal{U}$ if, and only if, either $b \in V\left(r_{7,1}\right) \backslash V\left(r_{1}\right)$ or $b \in V\left(r_{8,1}\right) \backslash V\left(r_{1}\right)$.

Next, we examine $V\left(r_{9}\right)$. Also, in this case, the general $b \in V\left(r_{9}\right)$ belongs to $\mathcal{U}$. The conditions that force $\operatorname{in}\left(r_{9}+J_{b}^{2}\right)$ to change are:

- $r_{9,1}=\left(b_{2}, b_{1}, b_{0}\right)$; 
- $r_{9,2}=\left(b_{3}, b_{2}, b_{1}, b_{0}\right)$.

Notice that $V\left(r_{9,2}\right) \subseteq V\left(r_{3}\right)$. We already studied $V\left(r_{3}\right)$, and so we stop the analysis of this case here because it does not give new insight. The general $b \in V\left(r_{9,1}\right)$ belongs to $\mathcal{U}$, but it appears as a new condition that forces $\operatorname{in}\left(r_{9,1}+J_{b}^{2}\right)$ to change, i.e., a level 3 condition. It is:

- $r_{9,1,1}=\left(b_{4}, b_{2}, b_{1}, b_{0}\right)$.

Trivially, $V\left(r_{9,1,1}\right) \subseteq V\left(r_{4}\right)$, which we studied above.

When studying $r_{10}$, no new condition shows up, and $V\left(r_{10}\right) \cap \mathcal{U} \neq \emptyset$.

The last codimension 2 ideal is $r_{11}$. Also, for it, we have that $V\left(r_{11}\right) \cap \mathcal{U} \neq \emptyset$, for that same argument, and the special condition is:

- $r_{11,1}=\left(b_{5}, b_{3}, b_{0}\right)$.

Studying $r_{11,1}$, the following two new conditions appear

- $r_{11,1,1}=\left(b_{5}, b_{3}, b_{1}, b_{0}\right)$

- $r_{11,1,2}=\left(b_{5}, b_{4}, b_{3}, b_{0}\right)$.

The first one defines a subvariety of $V\left(r_{3}\right)$, while the second one defines a subvariety of $V\left(r_{2}\right)$, and so none of them has to be studied. Moreover, the points outside $V\left(r_{11,1,1}\right) \cup V\left(r_{11,1,2}\right)$ are in $\mathcal{U}$.

Now, we consider the codimension 1 ideals.

The first one we consider is $r_{12}$. For the general $b \in V\left(r_{12}\right), A^{H, b}$ is unobstructed and so $b \in \mathcal{U}$. The new conditions forcing the initial ideal to change are:

$$
\begin{aligned}
& \text { - } r_{12,1}=\left(-b_{1}^{2}+b_{0} b_{2}, b_{3}\right) \\
& \text { - } r_{12,2}=\left(b_{0}, b_{3}\right) ; \\
& \text { - } r_{12,3}=\left(-b_{0} b_{4}^{2}-b_{1}^{2} b_{5}+b_{0} b_{2} b_{5}, b_{3}\right) .
\end{aligned}
$$

There exists $b \in V\left(r_{12,2}\right) \cap \mathcal{U}$, and the only new condition is:

- $r_{12,2,1}=\left(b_{4}, b_{3}, b_{0}\right)$.

Such a case was already examined above because $V\left(r_{12,2,1}\right) \subseteq V\left(r_{8}\right)$.

When we take a general $b \in V\left(r_{12,1}\right)$, then again $b \in \mathcal{U}$. The only new condition to examine is: 
- $r_{12,1,1}=\left(b_{3}, b_{2}, b_{1}\right)$.

We can argue as above because $V\left(r_{12,1,1}\right) \subseteq V\left(r_{6}\right)$.

Finally, the scheme $A^{H, b}$ associated to the general $b \in V\left(r_{12,3}\right)$ is unobstructed, i.e., $b \in \mathcal{U}$. As no new condition shows up, the analysis of $r_{12}$ is finished.

Also, in the next case, the general $b \in V\left(r_{13}\right)$ is in $\mathcal{U}$. The conditions that force $\operatorname{in}\left(r_{13}+J^{2}\right)$ to change are:

- $r_{13,1}=\left(b_{2}, b_{1}\right)$;

- $r_{13,2}=\left(b_{1}, b_{0}\right)$;

- $r_{13,3}=\left(-b_{2} b_{3}^{2}-b_{0} b_{4}^{2}+b_{0} b_{2} b_{5}, b_{1}\right)$.

If $b$ is general in either $V\left(r_{13,1}\right)$ or $V\left(r_{13,3}\right)$, then $b \in \mathcal{U}$. Since no new condition appears it follows that the same holds for each $b$. Similarly, the general $b \in V\left(r_{13,2}\right)$ is in $\mathcal{U}$, but the following two new conditions show up:

- $r_{13,2,1}=\left(b_{4}, b_{1}, b_{0}\right)$;

- $r_{13,2,2}=\left(b_{2}^{3}-b_{4}^{3}+b_{2} b_{4} b_{5}, b_{3}, b_{1}, b_{0}\right)$.

The first ideal defines a subvariety of $V\left(r_{7}\right)$, while the second one defines a subvariety of $V\left(r_{3}\right)$, and so none of them has to be studied.

The analysis of $r_{14}$ is more difficult than the previous ones. We still have $V\left(r_{14}\right) \cap \mathcal{U} \neq \emptyset$, and the only new condition that appears is:

- $r_{14,1}=\left(b_{1} b_{2}^{2} b_{3}^{2}-2 b_{1}^{2} b_{2} b_{3} b_{4}+b_{1}^{3} b_{4}^{2}-b_{3}^{3} b_{4}^{2}+2 b_{1} b_{3}^{2} b_{4} b_{5}-b_{1}^{2} b_{3} b_{5}^{2},-b_{1}^{2}+\right.$ $\left.b_{0} b_{2}\right)$.

Again, $V\left(r_{14,1}\right) \cap \mathcal{U}$ is not empty, but a new condition appears:

- $r_{14,1,1}=\left(b_{2} b_{3}+b_{1} b_{4}, b_{1} b_{3}+b_{0} b_{4}, b_{1}^{2}-b_{0} b_{2}, 4 b_{2}^{3} b_{4}+b_{4}^{4}+2 b_{2} b_{4}^{2} b_{5}+\right.$ $b_{2}^{2} b_{5}^{2}, 4 b_{1} b_{2}^{2} b_{4}-b_{3} b_{4}^{3}+2 b_{1} b_{4}^{2} b_{5}+b_{1} b_{2} b_{5}^{2}, 4 b_{0} b_{2}^{2} b_{4}+b_{3}^{2} b_{4}^{2}+2 b_{0} b_{4}^{2} b_{5}+$ $b_{0} b_{2} b_{5}^{2}, 4 b_{0} b_{1} b_{2} b_{4}-b_{3}^{3} b_{4}-2 b_{0} b_{3} b_{4} b_{5}+b_{0} b_{1} b_{5}^{2}, b_{3}^{4}+4 b_{0}^{2} b_{2} b_{4}+$ $\left.2 b_{0} b_{3}^{2} b_{5}+b_{0}^{2} b_{5}^{2}\right)$.

although $V\left(r_{14,1,1}\right) \cap \mathcal{U} \neq \emptyset$, several non-generic new conditions show up:

- $r_{14,1,1,1}=\left(4 b_{2}^{3} b_{4}+b_{4}^{4}+2 b_{2} b_{4}^{2} b_{5}+b_{2}^{2} b_{5}^{2}, b_{3}, b_{1}, b_{0}\right)$;

- $r_{14,1,1,2}=\left(b_{3}+b_{4}, b_{1}-b_{2}, b_{0}-b_{2}, 4 b_{2}^{3} b_{4}+b_{4}^{4}+2 b_{2} b_{4}^{2} b_{5}+b_{2}^{2} b_{5}^{2}\right)$;

- $r_{14,1,1,3}=\left(b_{4}, b_{2}, b_{1}, b_{3}^{2}+b_{0} b_{5}\right)$; 
- $r_{14,1,1,4}=\left(b_{0}+b_{1}+b_{2}, b_{3}^{2}-b_{3} b_{4}+b_{4}^{2}, b_{2} b_{3}+b_{1} b_{4}, b_{1} b_{3}-b_{1} b_{4}-\right.$ $b_{2} b_{4}, b_{1}^{2}+b_{1} b_{2}+b_{2}^{2}, 4 b_{2}^{3} b_{4}+b_{4}^{4}+2 b_{2} b_{4}^{2} b_{5}+b_{2}^{2} b_{5}^{2}, 4 b_{1} b_{2}^{2} b_{4}-b_{3} b_{4}^{3}+$ $\left.2 b_{1} b_{4}^{2} b_{5}+b_{1} b_{2} b_{5}^{2}\right)$

- $r_{14,1,1,5}=\left(b_{3}, b_{1}, b_{0}, 3 b_{4}^{2}-b_{2} b_{5}, 9 b_{2} b_{4}+4 b_{5}^{2}, 3 b_{2}^{2}+4 b_{4} b_{5}\right)$;

- $r_{14,1,1,6}=\left(b_{4}+b_{5}, b_{3}, b_{2}-b_{5}, b_{1}, b_{0}\right)$;

- $r_{14,1,1,7}=\left(b_{4}^{2}-b_{4} b_{5}+b_{5}^{2}, b_{3}, b_{2}-b_{4}+b_{5}, b_{1}, b_{0}\right)$.

The ideals $r_{14,1,1,1}, r_{14,1,1,5}, r_{14,1,1,6}$ and $r_{14,1,1,7}$ define subvarieties of $V\left(r_{3}\right)$, while $r_{14,1,1,3}$ defines a subvariety of $V\left(r_{4}\right)$, and so we do not study them.

We have $V\left(r_{14,1,1,2}\right) \cap \mathcal{U} \neq \emptyset$, and the new non-general conditions are:

- $r_{14,1,1,2,1}=\left(b_{5}, b_{4}, b_{3}, b_{1}-b_{2}, b_{0}-b_{2}\right)$;

- $r_{14,1,1,2,2}=\left(b_{4}^{2}-b_{4} b_{5}+b_{5}^{2}, b_{2}-b_{4}+b_{5}, b_{3}+b_{4}, b_{1}-b_{2}, b_{0}-b_{2}\right)$;

- $r_{14,1,1,2,3}=\left(b_{4}+b_{5}, b_{2}+b_{4}, b_{3}+b_{4}, b_{1}-b_{2}, b_{0}-b_{2}\right)$.

The first ideal defines a subvariety of $V\left(r_{2}\right)$, and so we do not study it. The general $b$ in either $V\left(r_{14,1,1,2,2}\right)$ or $V\left(r_{14,1,1,2,3}\right)$ is in $\mathcal{U}$ and, in both cases, the non-general condition is $b_{0}=\cdots=b_{5}=0$ that defines a point in $V\left(r_{1}\right)$.

We have $V\left(r_{14,1,1,4}\right) \cap \mathcal{U} \neq \emptyset$, and the non-general conditions are:

- $r_{14,1,1,4,1}=\left(b_{4}^{2}-b_{4} b_{5}+b_{5}^{2}, b_{3}-b_{4}+b_{5}, b_{2}-b_{3}, b_{1}-b_{5}, b_{0}+b_{1}+b_{2}\right)$;

- $r_{14,1,1,4,2}=\left(b_{5}, b_{4}, b_{3}, b_{1}^{2}+b_{1} b_{2}+b_{2}^{2}, b_{0}+b_{1}+b_{2}\right)$;

- $r_{14,1,1,4,3}=\left(b_{4}^{2}-b_{4} b_{5}+b_{5}^{2}, b_{3}-b_{5}, b_{2}-b_{4}+b_{5}, b_{1}+b_{4}, b_{0}+b_{1}+b_{2}\right)$;

- $r_{14,1,1,4,4}=\left(b_{4}+b_{5}, b_{3}^{2}+b_{3} b_{5}+b_{5}^{2}, b_{2}+b_{4}, b_{1}-b_{3}, b_{0}+b_{1}+b_{2}\right)$.

$V\left(r_{14,1,1,4,2}\right) \subseteq V\left(r_{2}\right)$, and so we do not consider it. The general $b$ in either $V\left(r_{14,1,1,4,1}\right)$ or $V\left(r_{14,1,1,4,3}\right)$ or $V\left(r_{14,1,1,4,4}\right)$ is in $\mathcal{U}$, and the only non-general condition is $b_{0}=\cdots=b_{5}=0$ in all three cases. It was considered earlier, and so the analysis of $r_{14}$ is complete, too.

The general $b \in V\left(r_{15}\right)$ belongs to $\mathcal{U}$, and the only new non-general condition that appears from the study of $\operatorname{in}\left(r_{15}+J^{2}\right)$ is:

- $r_{15,1}=\left(b_{1} b_{3}-b_{0} b_{4}, b_{0} b_{2}^{2} b_{3}+b_{1}^{3} b_{4}-2 b_{0} b_{1} b_{2} b_{4}-b_{3}^{3} b_{4}+2 b_{0} b_{3} b_{4} b_{5}-\right.$ $\left.b_{0} b_{1} b_{5}^{2}\right)$.

Again, $V\left(r_{15,1}\right) \cap \mathcal{U} \neq \emptyset$, and no new non-general condition shows up. Hence, the analysis of $r_{15}$ is complete. 
$V\left(r_{16}\right) \cap \mathcal{U} \neq \emptyset$ and no new non-general condition appears. We infer $V\left(r_{16}\right) \backslash V\left(r_{1}\right) \subseteq \mathcal{U}$.

So, we can consider the last and more difficult case $r_{17}$. As in most of the previous cases, $V\left(r_{17}\right) \cap \mathcal{U} \neq \emptyset$, and the new non-general conditions are:

- $r_{17,1}=\left(b_{2} b_{3}^{2}-2 b_{1} b_{3} b_{4}+b_{0} b_{4}^{2}+b_{1}^{2} b_{5}-b_{0} b_{2} b_{5}, b_{1}^{3}-b_{0} b_{1} b_{2}-b_{3}^{3}+\right.$ $\left.b_{0} b_{3} b_{5}\right)$

- $r_{17,2}=\left(b_{1} b_{3}+b_{0} b_{4}, b_{0} b_{2}^{2} b_{3}-b_{1}^{3} b_{4}-2 b_{0} b_{1} b_{2} b_{4}+b_{3}^{3} b_{4}+2 b_{0} b_{3} b_{4} b_{5}-\right.$ $\left.b_{0} b_{1} b_{5}^{2}\right)$.

$r_{17,1}$ contains $r_{16}$, and so we can skip its study. However, $V\left(r_{17,2}\right) \cap \mathcal{U} \neq$ $\emptyset$. The new non-general conditions that show up from the study of $\operatorname{in}\left(r_{17,2}+J_{b}^{2}\right)$ are:

- $r_{17,2,1}=\left(b_{4}^{2}-b_{2} b_{5}, b_{3} b_{4}-b_{1} b_{5}, b_{3}^{2}+b_{0} b_{5}, b_{2} b_{3}-b_{1} b_{4}, b_{1} b_{3}+\right.$ $\left.b_{0} b_{4}, b_{1}^{2}+b_{0} b_{2}\right)$

- $r_{17,2,2}=\left(b_{5}, b_{4}, b_{3}, b_{1}^{2}+b_{0} b_{2}\right)$;

- $r_{17,2,3}=\left(b_{4}, b_{2}, b_{1}, b_{3}^{2}+b_{0} b_{5}\right)$;

- $r_{17,2,4}=\left(b_{3}, b_{1}, b_{0}, 4 b_{2}^{3} b_{4}-27 b_{4}^{4}+18 b_{2} b_{4}^{2} b_{5}+b_{2}^{2} b_{5}^{2}+4 b_{4} b_{5}^{3}\right)$;

- $r_{17,2,5}=\left(b_{3}, b_{1}, b_{0}, 4 b_{2}^{3} b_{4}-15 b_{4}^{4}+4 b_{2} b_{4}^{2} b_{5}+3 b_{2}^{2} b_{5}^{2}+4 b_{4} b_{5}^{3}\right)$;

- $r_{17,2,6}=\left(9 b_{4}^{2}-3 b_{4} b_{5}+b_{5}^{2}, b_{3}, b_{2}-3 b_{4}+b_{5}, b_{1}, b_{0}\right)$;

- $r_{17,2,7}=\left(3 b_{4}+b_{5}, b_{3}, b_{2}+3 b_{4}, b_{1}, b_{0}\right)$;

- $r_{17,2,8}=\left(b_{5}, b_{3}, b_{2}, b_{1}, b_{0}\right)$;

- $r_{17,2,9}=\left(b_{4}^{2}+b_{4} b_{5}+b_{5}^{2}, b_{3}, b_{2}+b_{4}+b_{5}, b_{0}, b_{1}\right)$;

- $r_{17,2,10}=\left(b_{4}-b_{5}, b_{3}, b_{2}-b_{4}, b_{0}, b_{1}\right)$;

- $r_{17,2,11}=\left(225 b_{4}^{3}+17 b_{5}^{3}, b_{3},-105 b_{4}^{2}+17 b_{2} b_{5}, 15 b_{2} b_{4}+7 b_{5}^{2}, 17 b_{2}^{2}+\right.$ $\left.49 b_{4} b_{5}, b_{0}, b_{1}\right)$;

- $r_{17,2,12}=\left(b_{1} b_{3}+b_{0} b_{4}, 3 b_{1}^{2} b_{4}+b_{0} b_{2} b_{4}-b_{3}^{2} b_{5}+b_{0} b_{5}^{2}, b_{2} b_{3}^{2}+3 b_{0} b_{4}^{2}+\right.$ $b_{1}^{2} b_{5}-b_{0} b_{2} b_{5}, b_{2}^{2} b_{3}-3 b_{1} b_{2} b_{4}+3 b_{3} b_{4} b_{5}-b_{1} b_{5}^{2}, b_{1}^{2} b_{2}-b_{0} b_{2}^{2}-3 b_{3}^{2} b_{4}-$ $b_{0} b_{4} b_{5}, b_{1}^{3}-b_{0} b_{1} b_{2}-b_{3}^{3}+b_{0} b_{3} b_{5}, 3 b_{2} b_{3} b_{4}^{2}-9 b_{1} b_{4}^{3}+4 b_{1} b_{2} b_{4} b_{5}+$ $b_{3} b_{4} b_{5}^{2}+b_{1} b_{5}^{3}, 4 b_{2}^{3} b_{4}-27 b_{4}^{4}+18 b_{2} b_{4}^{2} b_{5}+b_{2}^{2} b_{5}^{2}+4 b_{4} b_{5}^{3}, 4 b_{1} b_{2}^{2} b_{4}-$ $\left.9 b_{3} b_{4}^{3}+b_{2} b_{3} b_{4} b_{5}+3 b_{1} b_{4}^{2} b_{5}+b_{1} b_{2} b_{5}^{2}\right)$

- $r_{17,2,13}=\left(b_{1} b_{3}+b_{0} b_{4}, b_{0} b_{2}^{2} b_{3}-b_{1}^{3} b_{4}-2 b_{0} b_{1} b_{2} b_{4}+b_{3}^{3} b_{4}+\right.$ $2 b_{0} b_{3} b_{4} b_{5}-b_{0} b_{1} b_{5}^{2}, 4 b_{0} b_{1}^{3} b_{4}+12 b_{0}^{2} b_{1} b_{2} b_{4}+b_{2} b_{3}^{2} b_{4}^{2}+3 b_{0} b_{4}^{4}-b_{2}^{2} b_{3}^{2} b_{5}-$ $12 b_{0}^{2} b_{3} b_{4} b_{5}+b_{1}^{2} b_{4}^{2} b_{5}-4 b_{0} b_{2} b_{4}^{2} b_{5}+4 b_{0}^{2} b_{1} b_{5}^{2}-b_{1}^{2} b_{2} b_{5}^{2}+b_{0} b_{2}^{2} b_{5}^{2}, b_{2}^{2} b_{3}^{4}+$ $16 b_{0}^{3} b_{1} b_{2} b_{4}+12 b_{0}^{2} b_{3}^{3} b_{4}+6 b_{0} b_{2} b_{3}^{2} b_{4}^{2}+9 b_{0}^{2} b_{4}^{4}-8 b_{0}^{3} b_{3} b_{4} b_{5}+2 b_{3}^{4} b_{4} b_{5}+$ $8 b_{0} b_{1}^{2} b_{4}^{2} b_{5}+4 b_{0}^{3} b_{1} b_{5}^{2}+b_{1}^{4} b_{5}^{2}-2 b_{0} b_{1}^{2} b_{2} b_{5}^{2}+b_{0}^{2} b_{2}^{2} b_{5}^{2}+4 b_{0} b_{3}^{2} b_{4} b_{5}^{2}+$ $2 b_{0}^{2} b_{4} b_{5}^{3}, b_{2}^{3} b_{3}^{3}+12 b_{0}^{2} b_{2} b_{3}^{2} b_{4}+4 b_{0}^{3} b_{4}^{3}+2 b_{1}^{3} b_{4}^{3}+3 b_{0} b_{1} b_{2} b_{4}^{3}-5 b_{3}^{3} b_{4}^{3}-$ $4 b_{0}^{2} b_{1}^{2} b_{4} b_{5}+4 b_{0}^{3} b_{2} b_{4} b_{5}+b_{2} b_{3}^{3} b_{4} b_{5}-11 b_{0} b_{3} b_{4}^{3} b_{5}+2 b_{0} b_{2} b_{3} b_{4} b_{5}^{2}+$ 
$7 b_{0} b_{1} b_{4}^{2} b_{5}^{2}+b_{1}^{3} b_{5}^{3}-b_{0} b_{1} b_{2} b_{5}^{3}, b_{2}^{4} b_{3}^{2}-16 b_{0}^{2} b_{1} b_{2} b_{4}^{2}+b_{1}^{2} b_{2}^{2} b_{4}^{2}+2 b_{0} b_{2}^{3} b_{4}^{2}-$ $12 b_{0} b_{3}^{3} b_{4}^{2}-6 b_{2} b_{3}^{2} b_{4}^{3}-9 b_{0} b_{4}^{5}+4 b_{2}^{2} b_{3}^{2} b_{4} b_{5}+8 b_{0}^{2} b_{3} b_{4}^{2} b_{5}-6 b_{1}^{2} b_{4}^{3} b_{5}+$ $4 b_{0} b_{2} b_{4}^{3} b_{5}-4 b_{0}^{2} b_{1} b_{4} b_{5}^{2}+4 b_{1}^{2} b_{2} b_{4} b_{5}^{2}+b_{0} b_{2}^{2} b_{4} b_{5}^{2}+b_{3}^{2} b_{4}^{2} b_{5}^{2}+2 b_{0} b_{4}^{2} b_{5}^{3}+$ $b_{1}^{2} b_{5}^{4}, 4 b_{0}^{3} b_{1}^{3}+b_{1}^{6}-4 b_{0}^{4} b_{1} b_{2}+b_{0} b_{1}^{4} b_{2}-b_{0}^{2} b_{1}^{2} b_{2}^{2}-b_{0}^{3} b_{2}^{3}-12 b_{0}^{3} b_{3}^{3}+b_{3}^{6}-$ $4 b_{0}^{2} b_{2} b_{3}^{2} b_{4}-4 b_{0}^{3} b_{4}^{3}-4 b_{0}^{4} b_{3} b_{5}+b_{0} b_{3}^{4} b_{5}-4 b_{0}^{2} b_{1}^{2} b_{4} b_{5}-4 b_{0}^{3} b_{2} b_{4} b_{5}-$ $b_{0}^{2} b_{3}^{2} b_{5}^{2}-b_{0}^{3} b_{5}^{3}, b_{2}^{3} b_{3}^{2} b_{4}^{2}+12 b_{0}^{2} b_{2} b_{3} b_{4}^{3}-4 b_{0}^{2} b_{1} b_{4}^{4}+b_{1}^{2} b_{2} b_{4}^{4}+2 b_{0} b_{2}^{2} b_{4}^{4}-$ $3 b_{3}^{2} b_{4}^{5}-16 b_{0}^{2} b_{1} b_{2} b_{4}^{2} b_{5}-10 b_{0} b_{4}^{5} b_{5}+b_{2}^{2} b_{3}^{2} b_{4} b_{5}^{2}+12 b_{0}^{2} b_{3} b_{4}^{2} b_{5}^{2}-6 b_{1}^{2} b_{4}^{3} b_{5}^{2}+$ $6 b_{0} b_{2} b_{4}^{3} b_{5}^{2}-4 b_{0}^{2} b_{1} b_{4} b_{5}^{3}+4 b_{1}^{2} b_{2} b_{4} b_{5}^{3}+b_{3}^{2} b_{4}^{2} b_{5}^{3}+2 b_{0} b_{4}^{2} b_{5}^{4}+b_{1}^{2} b_{5}^{5}$, $4 b_{0} b_{1}^{2} b_{2}^{2} b_{4}^{2}-4 b_{0}^{2} b_{2}^{3} b_{4}^{2}-4 b_{0} b_{2} b_{3}^{2} b_{4}^{3}-b_{2}^{2} b_{3} b_{4}^{4}+3 b_{1} b_{2} b_{4}^{5}+2 b_{2}^{3} b_{3} b_{4}^{2} b_{5}+$ $4 b_{0} b_{1}^{2} b_{4}^{3} b_{5}+16 b_{0}^{2} b_{2} b_{4}^{3} b_{5}-6 b_{1} b_{2}^{2} b_{4}^{3} b_{5}-3 b_{3} b_{4}^{5} b_{5}-b_{2}^{4} b_{3} b_{5}^{2}+3 b_{1} b_{2}^{3} b_{4} b_{5}^{2}+$ $12 b_{0} b_{3}^{2} b_{4}^{2} b_{5}^{2}+6 b_{2} b_{3} b_{4}^{3} b_{5}^{2}+b_{1} b_{4}^{4} b_{5}^{2}-3 b_{2}^{2} b_{3} b_{4} b_{5}^{3}+4 b_{0}^{2} b_{4}^{2} b_{5}^{3}-2 b_{1} b_{2} b_{4}^{2} b_{5}^{3}+$ $b_{1} b_{2}^{2} b_{5}^{4}, 16 b_{0}^{2} b_{1} b_{2}^{2} b_{4}^{2}+b_{2}^{2} b_{3}^{2} b_{4}^{3}-4 b_{0}^{2} b_{3} b_{4}^{4}-3 b_{1}^{2} b_{4}^{5}+2 b_{0} b_{2} b_{4}^{5}-b_{2}^{3} b_{3}^{2} b_{4} b_{5}-$ $12 b_{0}^{2} b_{2} b_{3} b_{4}^{2} b_{5}-4 b_{0}^{2} b_{1} b_{4}^{3} b_{5}+7 b_{1}^{2} b_{2} b_{4}^{3} b_{5}-2 b_{0} b_{2}^{2} b_{4}^{3} b_{5}+b_{3}^{2} b_{4}^{4} b_{5}+$ $4 b_{0}^{2} b_{1} b_{2} b_{4} b_{5}^{2}-4 b_{1}^{2} b_{2}^{2} b_{4} b_{5}^{2}-b_{2} b_{3}^{2} b_{4}^{2} b_{5}^{2}+2 b_{0} b_{4}^{4} b_{5}^{2}+b_{1}^{2} b_{4}^{2} b_{5}^{3}-2 b_{0} b_{2} b_{4}^{2} b_{5}^{3}-$ $b_{1}^{2} b_{2} b_{5}^{4}, 48 b_{0}^{4} b_{2} b_{3} b_{4}-4 b_{0} b_{2} b_{3}^{4} b_{4}-16 b_{0}^{4} b_{1} b_{4}^{2}+48 b_{0}^{3} b_{2}^{2} b_{4}^{2}-3 b_{2}^{2} b_{3}^{3} b_{4}^{2}-$ $6 b_{0} b_{2} b_{3} b_{4}^{4}-9 b_{0} b_{1} b_{4}^{5}+48 b_{0}^{3} b_{3}^{3} b_{5}-4 b_{3}^{6} b_{5}+8 b_{0}^{2} b_{2} b_{3}^{2} b_{4} b_{5}-32 b_{0}^{3} b_{4}^{3} b_{5}+$ $24 b_{0} b_{1} b_{2} b_{4}^{3} b_{5}+6 b_{3}^{3} b_{4}^{3} b_{5}+16 b_{0}^{4} b_{3} b_{5}^{2}-4 b_{0} b_{3}^{4} b_{5}^{2}+16 b_{0}^{2} b_{1}^{2} b_{4} b_{5}^{2}+$ $12 b_{0}^{3} b_{2} b_{4} b_{5}^{2}-12 b_{0} b_{1} b_{2}^{2} b_{4} b_{5}^{2}+15 b_{0} b_{3} b_{4}^{3} b_{5}^{2}+4 b_{0}^{2} b_{3}^{2} b_{5}^{3}-12 b_{0} b_{1} b_{4}^{2} b_{5}^{3}+$ $4 b_{0}^{3} b_{5}^{4}-3 b_{1}^{3} b_{5}^{4}, 12 b_{0}^{3} b_{2} b_{3}^{3}-b_{2} b_{3}^{6}+4 b_{0}^{4} b_{3} b_{4}^{2}-3 b_{0} b_{3}^{4} b_{4}^{2}+4 b_{0}^{4} b_{2} b_{3} b_{5}-$ $b_{0} b_{2} b_{3}^{4} b_{5}+4 b_{0}^{4} b_{1} b_{4} b_{5}-7 b_{0}^{2} b_{3}^{2} b_{4}^{2} b_{5}+b_{0}^{2} b_{2} b_{3}^{2} b_{5}^{2}-5 b_{0}^{3} b_{4}^{2} b_{5}^{2}-b_{0}^{2} b_{1}^{2} b_{5}^{3}+$ $b_{0}^{3} b_{2} b_{5}^{3}, 8 b_{1}^{4} b_{4}^{4}+28 b_{0} b_{1}^{2} b_{2} b_{4}^{4}-4 b_{0}^{2} b_{2}^{2} b_{4}^{4}-4 b_{0} b_{3}^{2} b_{4}^{5}-3 b_{2} b_{3} b_{4}^{6}+9 b_{1} b_{4}^{7}+$ $16 b_{0}^{2} b_{2}^{3} b_{4}^{2} b_{5}+16 b_{0} b_{2} b_{3}^{2} b_{4}^{3} b_{5}+6 b_{2}^{2} b_{3} b_{4}^{4} b_{5}+32 b_{0}^{2} b_{4}^{5} b_{5}-22 b_{1} b_{2} b_{4}^{5} b_{5}-$ $3 b_{2}^{3} b_{3} b_{4}^{2} b_{5}^{2}+4 b_{0} b_{1}^{2} b_{4}^{3} b_{5}^{2}-4 b_{0}^{2} b_{2} b_{4}^{3} b_{5}^{2}+17 b_{1} b_{2}^{2} b_{4}^{3} b_{5}^{2}-b_{3} b_{4}^{5} b_{5}^{2}+4 b_{0}^{2} b_{2}^{2} b_{4} b_{5}^{3}-$ $4 b_{1} b_{2}^{3} b_{4} b_{5}^{3}+4 b_{0} b_{3}^{2} b_{4}^{2} b_{5}^{3}+2 b_{2} b_{3} b_{4}^{3} b_{5}^{3}-b_{1} b_{4}^{4} b_{5}^{3}-b_{2}^{2} b_{3} b_{4} b_{5}^{4}-4 b_{0}^{2} b_{4}^{2} b_{5}^{4}+$ $2 b_{1} b_{2} b_{4}^{2} b_{5}^{4}-b_{1} b_{2}^{2} b_{5}^{5}, 16 b_{0}^{2} b_{2}^{4} b_{4}^{2}-16 b_{3}^{4} b_{4}^{4}-28 b_{0} b_{1}^{2} b_{4}^{5}-64 b_{0}^{2} b_{2} b_{4}^{5}-$ $4 b_{1} b_{2}^{2} b_{4}^{5}+9 b_{3} b_{4}^{7}-4 b_{0} b_{1}^{2} b_{2} b_{4}^{3} b_{5}-4 b_{0}^{2} b_{2}^{2} b_{4}^{3} b_{5}+8 b_{1} b_{2}^{3} b_{4}^{3} b_{5}-68 b_{0} b_{3}^{2} b_{4}^{4} b_{5}-$ $19 b_{2} b_{3} b_{4}^{5} b_{5}-3 b_{1} b_{4}^{6} b_{5}+4 b_{0}^{2} b_{2}^{3} b_{4} b_{5}^{2}-4 b_{1} b_{2}^{4} b_{4} b_{5}^{2}+4 b_{0} b_{2} b_{3}^{2} b_{4}^{2} b_{5}^{2}+$ $11 b_{2}^{2} b_{3} b_{4}^{3} b_{5}^{2}-28 b_{0}^{2} b_{4}^{4} b_{5}^{2}+5 b_{1} b_{2} b_{4}^{4} b_{5}^{2}-b_{2}^{3} b_{3} b_{4} b_{5}^{3}-4 b_{0}^{2} b_{2} b_{4}^{2} b_{5}^{3}-$ $b_{1} b_{2}^{2} b_{4}^{2} b_{5}^{3}-b_{1} b_{2}^{3} b_{5}^{4}, 768 b_{0}^{4} b_{1} b_{2} b_{4}^{2}+128 b_{0}^{3} b_{2}^{3} b_{4}^{2}+16 b_{1}^{3} b_{2}^{3} b_{4}^{2}+32 b_{0} b_{1} b_{2}^{4} b_{4}^{2}+$ $576 b_{0}^{3} b_{3}^{3} b_{4}^{2}-48 b_{3}^{6} b_{4}^{2}+320 b_{0}^{2} b_{2} b_{3}^{2} b_{4}^{3}+416 b_{0}^{3} b_{4}^{5}-68 b_{1}^{3} b_{4}^{5}-192 b_{0} b_{1} b_{2} b_{4}^{5}+$ $4 b_{2}^{3} b_{4}^{5}+8 b_{3}^{3} b_{4}^{5}-27 b_{4}^{8}-384 b_{0}^{4} b_{3} b_{4}^{2} b_{5}-1088 b_{0}^{3} b_{2} b_{4}^{3} b_{5}+16 b_{1}^{3} b_{2} b_{4}^{3} b_{5}+$ $104 b_{0} b_{1} b_{2}^{2} b_{4}^{3} b_{5}-8 b_{2}^{4} b_{4}^{3} b_{5}+112 b_{2} b_{3}^{3} b_{4}^{3} b_{5}+392 b_{0} b_{3} b_{4}^{5} b_{5}+72 b_{2} b_{4}^{6} b_{5}+$ $192 b_{0}^{4} b_{1} b_{4} b_{5}^{2}-160 b_{0}^{3} b_{2}^{2} b_{4} b_{5}^{2}+4 b_{1}^{3} b_{2}^{2} b_{4} b_{5}^{2}-8 b_{0} b_{1} b_{2}^{3} b_{4} b_{5}^{2}+4 b_{2}^{5} b_{4} b_{5}^{2}-$ $88 b_{2}^{2} b_{3}^{3} b_{4} b_{5}^{2}-1120 b_{0}^{2} b_{3}^{2} b_{4}^{2} b_{5}^{2}-408 b_{0} b_{2} b_{3} b_{4}^{3} b_{5}^{2}+20 b_{0} b_{1} b_{4}^{4} b_{5}^{2}-62 b_{2}^{2} b_{4}^{4} b_{5}^{2}-$ $192 b_{0}^{3} b_{4}^{2} b_{5}^{3}+176 b_{1}^{3} b_{4}^{2} b_{5}^{3}+160 b_{0} b_{1} b_{2} b_{4}^{2} b_{5}^{3}+16 b_{2}^{3} b_{4}^{2} b_{5}^{3}-128 b_{3}^{3} b_{4}^{2} b_{5}^{3}+$ $4 b_{4}^{5} b_{5}^{3}-48 b_{0}^{2} b_{1}^{2} b_{5}^{4}-48 b_{1}^{3} b_{2} b_{5}^{4}+44 b_{0} b_{1} b_{2}^{2} b_{5}^{4}+b_{2}^{4} b_{5}^{4}-272 b_{0} b_{3} b_{4}^{2} b_{5}^{4}-$ $\left.8 b_{2} b_{4}^{3} b_{5}^{4}+128 b_{0} b_{1} b_{4} b_{5}^{5}+4 b_{2}^{2} b_{4} b_{5}^{5}\right)$. 
We have $V\left(r_{17,2, j}\right) \subseteq V\left(r_{3}\right), j=4, \ldots, 11, V\left(r_{17,2,1}\right) \subseteq V\left(r_{10}\right)$, $V\left(r_{17,2,2}\right) \subseteq V\left(r_{2}\right), V\left(r_{17,2,3}\right) \subseteq V\left(r_{4}\right)$, and finally, $V\left(r_{17,2,12}\right) \subseteq$ $V\left(r_{16}\right)$. Hence, the only ideal to be studied is $r_{17,2,13}$.

Again, the general $b \in V\left(r_{17,2,13}\right)$ is in $\mathcal{U}$, but many new non-general conditions appear. They are:

- $r_{17,2,13,1}=\left(3 b_{4}+b_{5}, b_{3}, b_{2}-b_{5}, b_{1}, b_{0}\right)$;

- $r_{17,2,13,2}=\left(4 b_{0}^{3} b_{1}+b_{1}^{4}+2 b_{0} b_{1}^{2} b_{2}+b_{0}^{2} b_{2}^{2}, b_{5}, b_{4}, b_{3}\right)$;

- $r_{17,2,13,3}=\left(12 b_{0}^{3} b_{3}^{3}-b_{3}^{6}+4 b_{0}^{4} b_{3} b_{5}-b_{0} b_{3}^{4} b_{5}+b_{0}^{2} b_{3}^{2} b_{5}^{2}+b_{0}^{3} b_{5}^{3}, b_{4}, b_{2}, b_{1}\right)$;

- $r_{17,2,13,4}=\left(b_{4}-b_{5}, b_{2}-b_{5}, b_{1}-b_{3}, b_{3}^{2}+b_{0} b_{5}\right)$;

- $r_{17,2,13,5}=\left(b_{2}+b_{4}+b_{5}, b_{4}^{2}+b_{4} b_{5}+b_{5}^{2}, b_{3} b_{4}-b_{1} b_{5}, b_{1} b_{4}+b_{1} b_{5}+\right.$ $\left.b_{3} b_{5}, b_{3}^{2}+b_{0} b_{5}, b_{1} b_{3}+b_{0} b_{4}, b_{1}^{2}-b_{0} b_{4}-b_{0} b_{5}\right)$

- $r_{17,2,13,6}=\left(b_{4}^{2}-b_{4} b_{5}+b_{5}^{2}, b_{3}, b_{2}-b_{4}+b_{5}, b_{1}, b_{0}\right)$;

- $r_{17,2,13,7}=\left(b_{4}+b_{5}, b_{3}, b_{2}+b_{4}, b_{1}, b_{0}\right)$;

- $r_{17,2,13,8}=\left(b_{3}+b_{4}, b_{1}-b_{2}, b_{0}-b_{2}, 4 b_{2}^{3} b_{4}+b_{4}^{4}+2 b_{2} b_{4}^{2} b_{5}+b_{2}^{2} b_{5}^{2}\right)$;

- $r_{17,2,13,9}=\left(b_{0}+b_{1}+b_{2}, b_{3}^{2}-b_{3} b_{4}+b_{4}^{2}, b_{2} b_{3}+b_{1} b_{4}, b_{1} b_{3}-b_{1} b_{4}-\right.$ $b_{2} b_{4}, b_{1}^{2}+b_{1} b_{2}+b_{2}^{2}, 4 b_{2}^{3} b_{4}+b_{4}^{4}+2 b_{2} b_{4}^{2} b_{5}+b_{2}^{2} b_{5}^{2}, 4 b_{1} b_{2}^{2} b_{4}-b_{3} b_{4}^{3}+$ $\left.2 b_{1} b_{4}^{2} b_{5}+b_{1} b_{2} b_{5}^{2}\right)$

- $r_{17,2,13,10}=\left(b_{5}, b_{4}, b_{3}, b_{1}+b_{2}, b_{0}-b_{2}\right)$;

- $r_{17,2,13,11}=\left(b_{5}, b_{4}, b_{3}, b_{1}^{2}-b_{1} b_{2}+b_{2}^{2}, b_{0}-b_{1}+b_{2}\right)$;

- $r_{17,2,13,12}=\left(4 b_{3}^{3}+3 b_{4}^{3}-2 b_{4}^{2} b_{5}-b_{4} b_{5}^{2}, b_{2}-b_{5}, b_{1}-b_{3}, b_{3}^{2}+\right.$ $\left.b_{0} b_{4}, 4 b_{0} b_{3}-3 b_{4}^{2}+2 b_{4} b_{5}+b_{5}^{2}\right)$

- $r_{17,2,13,13}=\left(b_{2} b_{3}+b_{1} b_{5}+b_{3} b_{5}, b_{1} b_{3}+b_{0} b_{4}, b_{2}^{2}+b_{2} b_{5}+b_{5}^{2}, b_{1} b_{2}-\right.$ $b_{3} b_{5}, b_{1}^{2}+b_{3}^{2}-b_{0} b_{4}, 4 b_{0} b_{1}+4 b_{0} b_{3}+2 b_{2} b_{4}+3 b_{4}^{2}-b_{2} b_{5}+2 b_{4} b_{5}, 4 b_{0}^{2} b_{3}-$ $\left.3 b_{3}^{2} b_{4}+3 b_{0} b_{4}^{2}-2 b_{3}^{2} b_{5}+b_{0} b_{5}^{2}\right)$.

The only ideals to be studied are $r_{17,2,13,12}$ and $r_{17,2,13,13}$ because $V\left(r_{17,2,13, j}\right)$ is contained in either $V\left(r_{2}\right)$ (in the cases $j=2,10,11$ ), or $V\left(r_{3}\right)$ (in the cases $\left.j=1,6,7\right)$, or $V\left(r_{16}\right)$ (in the cases $j=4,5$ ), or $V\left(r_{14}\right)$ (in the cases $\left.j=8,9\right)$.

For $b$ general, in either $V\left(r_{17,2,13,12}\right)$ or $V\left(r_{17,2,13,13}\right)$, then $b \in \mathcal{U}$. The new non-general conditions inside $r_{17,2,13,12}$ are:

- $r_{17,2,13,12,1}=\left(b_{4}+b_{5}, b_{3}-b_{5}, b_{0}-b_{5}, b_{2}-b_{5}, b_{1}-b_{3}\right)$;

- $r_{17,2,13,12,2}=\left(b_{4}+b_{5}, b_{3}^{2}+b_{3} b_{5}+b_{5}^{2}, b_{0}+b_{3}+b_{5}, b_{2}-b_{5}, b_{1}-b_{3}\right)$,

but both ideals define subvarieties of $V\left(r_{14}\right)$, and so we do not study them.

The new non-general conditions inside $r_{17,2,13,13}$ are: 
- $r_{17,2,13,13,1}=\left(b_{4}^{2}-b_{4} b_{5}+b_{5}^{2}, b_{3}+b_{4}, b_{2}-b_{4}+b_{5}, b_{1}-b_{4}+b_{5}, b_{0}-\right.$ $\left.b_{4}+b_{5}\right)$

- $r_{17,2,13,13,2}=\left(b_{4}^{2}-b_{4} b_{5}+b_{5}^{2}, b_{3}-b_{4}+b_{5}, b_{2}-b_{4}+b_{5}, b_{1}-b_{5}, b_{0}+\right.$ $\left.b_{4}\right)$;

- $r_{17,2,13,13,3}=\left(b_{4}^{2}-b_{4} b_{5}+b_{5}^{2}, b_{3}-b_{5}, b_{2}-b_{4}+b_{5}, b_{1}+b_{4}, b_{0}-b_{5}\right)$,

but they all define subvarieties of $V\left(r_{14}\right)$, and so they are not to be studied. Hence, the analysis of $r_{17}$ is over.

Our analysis in now complete when $H=y_{2}^{3}+y_{3}^{3}+y_{4}^{3}$.

Let $H=y_{2}^{3}+y_{3}^{2} y_{4}$. $\operatorname{Ann}\left(F^{H, b}\right)$ is generated by

$$
\begin{gathered}
12 x_{4}^{2}-b_{5} x_{1}^{3}, \quad 12 x_{4} x_{2}-b_{3} x_{1}^{3}, \quad 12 x_{3} x_{2}-b_{1} x_{1}^{3}, \\
3 x_{2} x_{1}-b_{0} p_{1}-3 b_{1} p_{2}-3 b_{3} p_{3}, \quad 3 x_{3} x_{1}-b_{1} p_{1}-3 b_{2} p_{2}-3 b_{4} p_{3}, \\
3 x_{4} x_{1}-b_{3} p_{1}-3 b_{4} p_{2}-3 b_{5} p_{3}, \quad x_{2} x_{1}^{2}, \quad 12 x_{2}^{2} x_{1}-b_{0} x_{1}^{4}, \quad 4 x_{2}^{3}-x_{1}^{4}, \\
x_{3} x_{1}^{2}, \quad 12 x_{3} x_{2} x_{1}-b_{1} x_{1}^{4}, \quad x_{3} x_{2}^{2}, \quad 12 x_{3}^{2} x_{1}-b_{2} x_{1}^{4}, \quad x_{3}^{2} x_{2}, \quad x_{3}^{3}, \\
x_{4} x_{1}^{2}, \quad 12 x_{4} x_{2} x_{1}-b_{3} x_{1}^{4}, \quad x_{4} x_{2}^{2}, \quad 12 x_{4} x_{3} x_{1}-b_{4} x_{1}^{4}, \quad x_{4} x_{3} x_{2}, \\
12 x_{4} x_{3}^{2}-x_{1}^{4}, \quad 12 x_{4}^{2} x_{1}-b_{5} x_{1}^{4}, \quad x_{4}^{2} x_{2}, \quad x_{4}^{2} x_{3}, \quad x_{4}^{3},
\end{gathered}
$$

where

$$
p_{1}=x_{2}^{2}-b_{0} x_{1}^{3} / 12, \quad p_{2}=x_{4} x_{3}-b_{4} x_{1}^{3} / 12, \quad p_{3}=x_{3}^{2}-b_{2} x_{1}^{3} / 12 .
$$

Let $H=y_{2}^{2} y_{3}+y_{3}^{2} y_{4}$. $\operatorname{Ann}\left(F^{H, b}\right)$ is generated by

$$
\begin{gathered}
12 x_{4}^{2}-b_{5} x_{1}^{3}, \quad 12 x_{4} x_{2}-b_{3} x_{1}^{3}, \quad x_{2} x_{1}-b_{0} p_{1}-b_{1} p_{2}-b_{3} p_{3}, \\
x_{2}^{2}-b_{0} x_{1}^{3} / 12-p_{2}, \quad x_{3} x_{1}-b_{1} p_{1}-b_{2} p_{2}-b_{4} p_{3}, \\
x_{4} x_{1}-b_{3} p_{1}-b_{4} p_{2}-b_{5} p_{3}, \quad x_{2} x_{1}^{2}, \quad 12 x_{2}^{2} x_{1}-b_{0} x_{1}^{4}, \\
x_{2}^{3}, \quad x_{3} x_{1}^{2}, \quad 12 x_{3} x_{2} x_{1}-b_{1} x_{1}^{4}, \quad 12 x_{3} x_{2}^{2}-x_{1}^{4}, \quad 12 x_{3}^{2} x_{1}-b_{2} x_{1}^{4}, \\
x_{3}^{2} x_{2}, \quad x_{3}^{3}, \quad x_{4} x_{1}^{2}, \quad 12 x_{4} x_{2} x_{1}-b_{3} x_{1}^{4}, \quad x_{4} x_{2}^{2}, \quad 12 x_{4} x_{3} x_{1}-b_{4} x_{1}^{4}, \\
x_{4} x_{3} x_{2}, \quad 12 x_{4} x_{3}^{2}-x_{1}^{4}, \quad 12 x_{4}^{2} x_{1}-b_{5} x_{1}^{4}, \quad x_{4}^{2} x_{2}, \quad x_{4}^{2} x_{3}, \quad x_{4}^{3},
\end{gathered}
$$

where

$$
p_{1}=x_{3} x_{2}-b_{1} x_{1}^{3} / 12, \quad p_{2}=x_{4} x_{3}-b_{4} x_{1}^{3} / 12, \quad p_{3}=x_{3}^{2}-b_{2} x_{1}^{3} / 12 .
$$

Let $H=y_{3}^{2} y_{4}-y_{3} y_{4}^{2}$. $b_{0}, b_{1}$ and $b_{3}$ cannot vanish simultaneously. 
$\operatorname{Ann}\left(F^{H, b}\right)$ is generated by

$$
\begin{gathered}
12 x_{2}^{2}-b_{0} x_{1}^{3}, \quad 12 x_{3} x_{2}-b_{1} x_{1}^{3}, \quad 12 x_{4} x_{2}-b_{3} x_{1}^{3}, \\
12 x_{3}^{2}+12 x_{4} x_{3}+12 x_{4}^{2}-\left(b_{2}+b_{4}+b_{5}\right) x_{1}^{3}, \quad b_{1} p_{1}-b_{0} p_{2}, \\
b_{3} p_{1}-b_{0} p_{3}, \quad b_{3} p_{2}-b_{1} p_{3}, \quad x_{4}^{3}, \quad 12 x_{4}^{2} x_{3}+x_{1}^{4}, x_{4}^{2} x_{2}, \quad 12 x_{4}^{2} x_{1}-b_{5} x_{1}^{4}, \\
12 x_{4} x_{3}^{2}-x_{1}^{4}, \quad x_{4} x_{3} x_{2}, \quad 12 x_{4} x_{3} x_{1}-b_{4} x_{1}^{4}, \quad x_{4} x_{2}^{2}, \quad 12 x_{4} x_{2} x_{1}-b_{3} x_{1}^{4}, \\
x_{3}^{3}, \quad x_{3}^{2} x_{2}, \quad 12 x_{3}^{2} x_{1}-b_{2} x_{1}^{4}, \quad x_{3} x_{2}^{2}, \quad 12 x_{3} x_{2} x_{1}-b_{1} x_{1}^{4}, \quad x_{2}^{3}, \\
12 x_{2}^{2} x_{1}-b_{0} x_{1}^{4}, \quad x_{4} x_{1}^{2}, \quad x_{3} x_{1}^{2}, \quad x_{2} x_{1}^{2},
\end{gathered}
$$

where

$$
\begin{aligned}
& p_{1}=x_{2} x_{1}+b_{1}\left(x_{4}^{2}-b_{5} x_{1}^{3} / 12\right)-b_{3}\left(x_{3}^{2}-b_{2} x_{1}^{3} / 12\right), \\
& p_{2}=x_{3} x_{1}+b_{2}\left(x_{4}^{2}-b_{5} x_{1}^{3} / 12\right)-b_{4}\left(x_{3}^{2}-b_{2} x_{1}^{3} / 12\right), \\
& p_{3}=x_{4} x_{1}+b_{4}\left(x_{4}^{2}-b_{5} x_{1}^{3} / 12\right)-b_{5}\left(x_{3}^{2}-b_{2} x_{1}^{3} / 12\right) .
\end{aligned}
$$

Let $H=y_{3} y_{4}^{2} . \quad b_{0}, b_{1}$ and $b_{3}$ cannot vanish simultaneously, and $\operatorname{Ann}\left(F^{H, b}\right)$ is generated by

$$
\begin{gathered}
12 x_{2}^{2}-b_{0} x_{1}^{3}, \quad 12 x_{3} x_{2}-b_{1} x_{1}^{3}, \quad 12 x_{4} x_{2}-b_{3} x_{1}^{3}, \quad 12 x_{3}^{2}-b_{2} x_{1}^{3}, \\
b_{1} p_{1}-b_{0} p_{2}, \quad b_{3} p_{1}-b_{0} p_{3}, \quad b_{3} p_{2}-b_{1} p_{3}, \quad x_{4}^{3}, \quad 12 x_{4}^{2} x_{3}-x_{1}^{4}, \quad x_{4}^{2} x_{2}, \\
x_{4} x_{3}^{2}, \quad x_{4} x_{3} x_{2}, \quad x_{4} x_{2}^{2}, \quad x_{3}^{3}, \quad x_{3}^{2} x_{2}, \quad x_{3} x_{2}^{2}, \quad x_{2}^{3}, \quad 12 x_{4}^{2} x_{1}-b_{5} x_{1}^{4}, \\
12 x_{4} x_{3} x_{1}-b_{4} x_{1}^{4}, \quad 12 x_{4} x_{2} x_{1}-b_{3} x_{1}^{4}, \quad 12 x_{3}^{2} x_{1}-b_{2} x_{1}^{4}, \\
12 x_{3} x_{2} x_{1}-b_{1} x_{1}^{4}, \quad 12 x_{2}^{2} x_{1}-b_{0} x_{1}^{4}, \quad x_{4} x_{1}^{2}, \quad x_{3} x_{1}^{2}, \quad x_{2} x_{1}^{2},
\end{gathered}
$$

where

$$
\begin{aligned}
& p_{1}=x_{2} x_{1}-b_{1}\left(x_{4}^{2}-b_{5} x_{1}^{3} / 12\right)-b_{3}\left(x_{4} x_{3}-b_{4} x_{1}^{3} / 12\right), \\
& p_{2}=x_{3} x_{1}-b_{2}\left(x_{4}^{2}-b_{5} x_{1}^{3} / 12\right)-b_{4}\left(x_{4} x_{3}-b_{4} x_{1}^{3} / 12\right), \\
& p_{3}=x_{4} x_{1}-b_{4}\left(x_{4}^{2}-b_{5} x_{1}^{3} / 12\right)-b_{5}\left(x_{4} x_{3}-b_{4} x_{1}^{3} / 12\right) .
\end{aligned}
$$

Finally, let $H=y_{4}^{3}$. If the first two lines of $M_{b}$ would be proportional, a suitable linear transformation on $y_{2}$ and $y_{3}$ would yield $b_{0}=b_{1}=$ $b_{3}=0$, whence $x_{2} \circ\left(y_{1} Q_{b}+H\right)=0$. Thus, $b \notin B_{H} \cdot \operatorname{Ann}\left(F^{H, b}\right)$ is 
generated by:

$$
\begin{gathered}
b_{6}\left(x_{4}^{2}-b_{5} x_{1}^{3} / 12\right)-3 x_{4} x_{1}\left(b_{1}^{2}-b_{0} b_{2}\right)+ \\
+3 x_{3} x_{1}\left(b_{1} b_{3}-b_{0} b_{4}\right)-3 x_{2} x_{1}\left(b_{2} b_{3}-b_{1} b_{4}\right), \\
12 x_{2}^{2}-b_{0} x_{1}^{3}, \quad 12 x_{2} x_{3}-b_{1} x_{1}^{3}, \quad 12 x_{3}^{2}-b_{2} x_{1}^{3}, \quad 12 x_{4} x_{2}-b_{3} x_{1}^{3}, \\
12 x_{4} x_{3}-b_{4} x_{1}^{3}, \quad 4 x_{4}^{3}-x_{1}^{4}, \quad x_{4}^{2} x_{3}, \quad x_{4}^{2} x_{2}, \quad 12 x_{4}^{2} x_{1}-b_{5} x_{1}^{4}, \quad x_{4} x_{3}^{2}, \\
x_{4} x_{3} x_{2}, \quad 12 x_{4} x_{3} x_{1}-b_{4} x_{1}^{4}, \quad x_{4} x_{2}^{2}, \quad 12 x_{4} x_{2} x_{1}-b_{3} x_{1}^{4}, \quad x_{4} x_{1}^{2}, \quad x_{3}^{3}, \\
x_{3}^{2} x_{2}, \quad 12 x_{3}^{2} x_{1}-b_{2} x_{1}^{4}, \quad x_{3} x_{2}^{2}, \quad 12 x_{3} x_{2} x_{1}-b_{1} x_{1}^{4}, x_{3} x_{1}^{2}, \quad x_{2}^{3}, \\
12 x_{2}^{2} x_{1}-b_{0} x_{1}^{4}, x_{2} x_{1}^{2},
\end{gathered}
$$

where $b_{6}=-\operatorname{det}\left(M_{b}\right)$.

Now, we summarize the previous results by giving the proof of Theorem B stated in the introduction.

Proof of Theorem B. If $H=y_{2}^{3}+y_{3}^{3}+y_{4}^{3}+t y_{2} y_{3} y_{4}$, with $t\left(t^{3}-216\right) \neq$ $0, A^{H, b}$ is unobstructed for every $b \in \mathbb{A}^{6}$, by Proposition 5.4. If $t\left(t^{3}-216\right)=0, H$ is a Fermat cubic, and so it can be written as $y_{2}^{3}+y_{3}^{3}+y_{4}^{3}$ up to a suitable change of coordinates. Let $F=$ $y_{1}^{4}+y_{1} Q_{b}+y_{2}^{3}+y_{3}^{3}+y_{4}^{3}$. Then $A^{H, b}$ is obstructed if, and only if, $b_{1}=b_{3}=b_{4}=0$, by Proposition 5.6.

If $H$ is either $y_{2}^{3}+y_{3}^{3}+y_{2} y_{3} y_{4}, y_{2}^{3}+y_{2} y_{3} y_{4}$ or $y_{2} y_{3} y_{4}$, then $A^{H, b}$ is unobstructed for every $b \in \mathbb{A}^{6}$, by Proposition 5.4.

If $H$ is either $y_{2}^{3}+y_{2}^{2} y_{4}, y_{2}^{2} y_{3}+y_{3}^{2} y_{4}, y_{3}^{2} y_{4}-y_{3} y_{4}^{2}, y_{3} y_{4}^{2}$ or $y_{4}^{3}$, the result was proved in Proposition 5.6.

Finally, if $H=0$, the $A^{H, b}$ is obstructed for every $b \in \mathbb{A}^{6}$, by Proposition 5.1.

We end the section with an example.

Example 5.7. Let us consider the two polynomials

$$
F_{1}=y_{1}^{4}+y_{1}\left(y_{2}^{2}+2 y_{3} y_{4}\right)+y_{2}^{2} y_{3}+y_{3}^{2} y_{4}
$$

and

$$
F_{2}=y_{1}^{4}+y_{1}\left(y_{2}^{2}+2 y_{3} y_{4}+y_{4}^{2}\right)+y_{2}^{2} y_{3}+y_{3}^{2} y_{4}
$$


Their apolar ideals are

$$
\begin{aligned}
\operatorname{Ann}\left(F_{1}\right)= & \left(x_{4}^{2}, 12 x_{4} x_{3}-12 x_{4} x_{1}-x_{1}^{3}, x_{3}^{2}-x_{3} x_{1}, x_{4} x_{2},\right. \\
& \left.x_{3} x_{2}-x_{2} x_{1}, 12 x_{2}^{2}-12 x_{4} x_{1}-x_{1}^{3}, x_{4} x_{1}^{2}, x_{3} x_{1}^{2}, x_{2} x_{1}^{2}\right)
\end{aligned}
$$

and

$$
\begin{gathered}
\operatorname{Ann}\left(F_{2}\right)=\left(12 x_{4}^{2}-x_{1}^{3}, 12 x_{4} x_{3}-12 x_{4} x_{1}+12 x_{3} x_{1}-x_{1}^{3}, x_{3}^{2}-x_{3} x_{1},\right. \\
\left.x_{4} x_{2}, x_{3} x_{2}-x_{2} x_{1}, 12 x_{2}^{2}-12 x_{4} x_{1}+12 x_{3} x_{1}-x_{1}^{3}, x_{4} x_{1}^{2}, x_{2} x_{1}^{2}\right),
\end{gathered}
$$

respectively. By using Singular, we get that

$$
N_{S[4] / \operatorname{Ann}\left(F_{1}\right)}=49, \quad N_{S[4] / \operatorname{Ann}\left(F_{2}\right)}=44 .
$$

Thus, $S[4] / \operatorname{Ann}\left(F_{1}\right)$ is obstructed, while $S[4] / \operatorname{Ann}\left(F_{2}\right)$ is unobstructed.

These results are coherent with Theorem B. In fact, the coefficients of the quadratic polynomial $Q_{1}=y_{2}^{2}+2 y_{3} y_{4}$ give a point in $V\left(b_{0}-\right.$ $\left.b_{4}, b_{3}, b_{5}\right)$, the locus of the obstructed schemes for the given $H=$ $y_{2}^{2} y_{3}+y_{3}^{2} y_{4}$, while the coefficients of the quadratic polynomial $Q_{2}=$ $y_{2}^{2}+2 y_{3} y_{4}+y_{4}^{2}$ give a point not in $V\left(b_{0}-b_{4}, b_{3}, b_{5}\right)$, and so the associated scheme is unobstructed.

\section{Appendix.}

6.1. Singular session. In this appendix, we show how we got the ideals $r_{1}, \ldots, r_{17}$ in the proof of Proposition 5.6. By adapting it, one can study all the cases of the above-mentioned proposition. We do not report the outputs of the command lines, starting with the symbol > and ending with the semicolumn, not to use too much space, but we insert comments staring with the symbol • to help the reader when performing the session by himself.

Here is the Singular session.

- the polynomial we consider is $p=x 4+x(b(0) * y 2+2 * b(1) * y z+$ $b(2) * z 2++2 * b(3) * y w+2 * b(4) * z w+b(5) * w 2)+y^{3}+z^{3}+w^{3}$

$>$ LIB "primdec.lib";

$>\operatorname{ring} r=0,(w, z, y, x, b(0 . .5)),(d p(3), \operatorname{lp}(1), d p(6))$;

$>$ poly $p 1=y 2-b(0) * x 3 / 12$;

$>$ poly $p 2=z 2-b(2) * x 3 / 12$; 
$>$ poly $p 3=w 2-b(5) * x 3 / 12$;

$>$ ideal $i 1=3 x y-b(0) * p 1-b(1) * p 2-b(3) * p 3$,

. $3 x z-b(1) * p 1-b(2) * p 2-b(4) * p 3,12 y z-b(1) * x 3$,

$.3 x w-b(3) * p 1-b(4) * p 2-b(5) * p 3,12 y w-b(3) * x 3,12 z w-b(4) * x 3$;

$>$ ideal $i 2=x 2 y, 12 x y 2-b(0) * x 4,4 y 3-x 4, x 2 z, 12 x y z-b(1) * x 4, y 2 z$,

$.12 x z 2-b(2) * x 4, y z 2,4 z 3-x 4, x 2 w, 12 x y w-b(3) * x 4, y 2 w$,

$.12 x z w-b(4) * x 4, y z w, z 2 w, 12 x w 2-b(5) * x 4, y w 2, z w 2,4 w 3-x 4$;

$>$ ideal $i 3=x 5, x 3 y, x 2 y 2, x y 3, y 4, x 3 z, x 2 y z, x y 2 z, y 3 z, x 2 z 2, x y z 2$,

. $y 2 z 2, x z 3, y z 3, z 4, x 3 w, x 2 y w, x y 2 w, y 3 w, x 2 z w, x y z w, y 2 z w, x z 2 w$,

. $y z 2 w, z 3 w, x 2 w 2, x y w 2, y 2 w 2, x z w 2, y z w 2, z 2 w 2, x w 3, y w 3, z w 3, w 4$;

- we compute the apolar ideal of $p$ and its Gröbner basis

$>$ ideal $i p=i 1, i 2, i 3$;

$>i p=\operatorname{std}(i p)$;

- we compute the square of the ideal ip and its Gröbner basis

$>$ ideal $i i=i 1 * i 1, i 1 * i 2, i 1 * i 3, i 2 * i 2, i 2 * i 3, i 3 * i 3$;

$>i i=\operatorname{std}(i i)$;

- we compute the initial ideal of $i i$

$>$ ideal $m i=\operatorname{lead}(i i)$;

- generic conditions for the computation of the Hilbert function

$>$ ideal $b g=b(0)-1, b(1)-1, b(2)-1, b(3)-1, b(4)-1, b(5)-1$;

- computation of the Hilbert function for a general $b$

$>m m=m i[1 . .158], b g$;

$>\operatorname{hilb}(\operatorname{std}(m m))$;

- the output of the previous line is $(1,4,10,20,14,4,2)$. So the general apolar ideal is unobstructed

- the initial terms of the polynomials in $i i$ depending on the $b(i)$ 's are (numbers refer to the list of the generators of $i i$, and $M$ is a monomial in $k[b])$ :

$w^{5} M$ is initial term for the polynomials $152,153,154$,

$z^{5} M$ is initial term for the polynomials $146,147,148$, 
$y^{5} M$ is initial term for the polynomials $138,139,140$, $w^{4} x M$ is initial term for the polynomials $134,135,136$, $w^{4} M$ is initial term for the polynomials $128, \ldots, 133$, $w^{3} z M$ is initial term for the polynomials $124,125,126$, $w z^{3} M$ is initial term for the polynomials $119,120,121$, $z^{4} x M$ is initial term for the polynomials $115,116,117$, $z^{4} M$ is initial term for the polynomials $107, \ldots, 114$, $w^{3} y M$ is initial term for the polynomials $103,104,105$, $z^{3} y M$ is initial term for the polynomials $97,98,99$, $w y^{3} M$ is initial term for the polynomials $89, \ldots, 92$, $z y^{3} M$ is initial term for the polynomials $85,86,87$, $y^{4} x M$ is initial term for the polynomials $81,82,83$, $y^{4} M$ is initial term for the polynomials $69, \ldots, 80$, $w^{2} x^{2} M$ is initial term for the polynomials $65,66,67$, $w^{3} x M$ is initial term for the polynomials $58, \ldots, 64$, $w^{2} z x M$ is initial term for the polynomials $53, \ldots, 56$, $w z^{2} x M$ is initial term for the polynomials $38, \ldots, 51$, $z^{3} x^{2} M$ is initial term for the polynomials $34,35,36$, $z^{3} x M$ is initial term for the polynomials 33 , $w^{2} y x M$ is initial term for the polynomial 31, $w z y w M$ is initial term for the polynomials 29 , $y^{3} x^{2} M$ is initial term for the polynomials $22,23,24$, $w^{2} x^{3} M$ is initial term for the polynomials 19,20 , $z^{2} x^{3} M$ is initial term for the polynomials 15,16 , $y^{2} x^{3} M$ is initial term for the polynomials 10,11 , $w x^{5} M$ is initial term for the polynomial 8, $z x^{5} M$ is initial term for the polynomial 6 , $x^{7} M$ is initial term for the polynomials $1,2,3$

- e.g. computation of the relations associated to $x^{7}$

$>$ int $i$;

$>$ ideal $r_{1}$;

$>$ for $(i=1 ; i<=3 ; i=i+1)\left\{r_{1}=r_{1},\left(i i[i]-\operatorname{reduce}\left(i i[i], \operatorname{std}\left(x^{7}\right)\right)\right) /\right.$ $(x 7) ;\}$;

- analogous computations allow us to compute all the ideals from $r_{1}$ to $r_{17}$. 
Acknowledgments. The authors want to express their thanks to Joachim Jelisiejew and Pedro Macias Marques for some instructing conversations and helpful remarks. We warmly thank the referee for her/his comments, corrections and suggestions which allowed us to improve considerably the exposition.

\section{REFERENCES}

1. D.A. Cartwright, D. Erman, M. Velasco and B. Viray, Hilbert schemes of 8 point in $\mathbb{A}^{d}$, Alg. Num. Th. 3 (2009), 763-795.

2. G. Casnati, Isomorphism types of Artinian Gorenstein local algebras of multiplicity at most 9, Comm. Algebra 38 (2010), 2738-2761.

3. G. Casnati and R. Notari, On the Gorenstein locus of some punctual Hilbert schemes, J. Pure Appl. Alg. 213 (2009), 2055-2074.

4. , On the irreducibility and the singularities of the Gorenstein locus of the punctual Hilbert scheme of degree 10, J. Pure Appl. Alg. 215 (2011), 1243-1254.

5. , Irreducibility of the Gorenstein locus of the punctual Hilbert scheme of degree 11, J. Pure Appl. Alg. 218 (2014), 1635-1651.

6. W. Decker, G.M. Greuel, G. Pfister and H. Schönemann, Singular 3-1-3, A computer algebra system for polynomial computations, http:// www.singular.uni-kl.de, 2011.

7. J. Elias and M.E. Rossi, Isomorphism classes of short Gorenstein local rings via Macaulay's inverse system, Trans. Amer. Math. Soc. 364 (2012), 4589-4604.

8. , Analytic isomorphisms of compressed local algebras, Proc. Amer. Math. Soc. 143 (2015), 973-987.

9. J. Elias and G. Valla, Structure theorems for certain Gorenstein ideals, Special volume in honor of Melvin Hochster, Michigan Math. J. 57 (2008), 269-292.

10. , Isomorphism classes of certain Artinian Gorenstein algebras, Alg. Rep. Th. 14 (2011), 429-448.

11. J. Emsalem, Géométrie des points épais, Bull. Soc. Math. France 106 (1978), $399-416$.

12. R. Hartshorne, Algebraic geometry, G.T.M. 52, Springer, New York, 1977.

13. A. Iarrobino, Compressed algebras: Artin algebras having given socle degrees and maximal length, Trans. Amer. Math. Soc. 285 (1994), 337-378.

14. , Associated graded algebra of a Gorenstein Artin algebra, Mem. Amer. Math. Soc. 107 (1994), viii+115.

15. J. Jelisiejew, Deformations of zero-dimensional schemes and applications, arXiv:1307.8108 [math.AC].

16. G. Mazzola, The algebraic and geometric classification of associative algebras of dimension five, Manuscr. Math. 27 (1979), 81-101.

17. , Generic finite schemes and Hochschild cocycles, Comm. Math. Helv. 55 (1980), 267-293. 
18. B. Poonen, The moduli space of commutative algebras of finite rank, J. Europ. Math. Soc. 10 (2008), 817-836.

19. , Isomorphism types of commutative algebras of finite rank over an algebraically closed field, in Computational arithmetic geometry, Kristin E. Lauter and Kenneth A. Ribet, eds., Contemp. Math. 463, American Mathematical Society, Providence, RI, 2008.

20. J.D. Sally, Stretched Gorenstein rings, J. Lond. Math. Soc. 20 (1979), 19-26.

Dipartimento di Scienze Matematiche, Politecnico di Torino, corso Duca DEgli Abruzzi 24, 10129 Torino, ItAly

Email address: gianfranco.casnati@polito.it

Dipartimento di Matematica "Francesco Brioschi," Politecnico di Milano, via Bonardi 9, 20133 Milano, Italy

Email address: roberto.notari@polimi.it 